\title{
A constellation of SmallSats with synthetic tracking cameras to search for $90 \%$ of potentially hazardous near-Earth objects
}

\author{
Michael Shao, Slava G. Turyshev, Sara Spangelo, Thomas Werne, and Chengxing Zhai
}

\begin{abstract}
Jet Propulsion Laboratory, California Institute of Technology 4800 Oak Grove Drive, Pasadena, CA 91109-8099, USA e-mail: turyshev@jpl.nasa.gov
\end{abstract}

Received 29 September 2016/ Accepted 31 March 2017

\begin{abstract}
We present a new space mission concept that is capable of finding, detecting, and tracking $90 \%$ of near-Earth objects (NEO) with $H$ magnitude of $H \leq 22$ (i.e., $\sim 140 \mathrm{~m}$ in size) that are potentially hazardous to the Earth. The new mission concept relies on two emerging technologies: the technique of synthetic tracking and the new generation of small and capable interplanetary spacecraft. Synthetic tracking is a technique that de-streaks asteroid images by taking multiple fast exposures. With synthetic tracking, an $800 \mathrm{~s}$ observation with a $10 \mathrm{~cm}$ telescope in space can detect a moving object with apparent magnitude of 20.5 without losing sensitivity from streaking. We refer to NEOs with a minimum orbit intersection distance of $<0.002$ au as Earth-grazers (EGs), representing typical albedo distributions. We show that a constellation of six SmallSats (comparable in size to 9U CubeSats) equipped with $10 \mathrm{~cm}$ synthetic tracking cameras and evenly-distributed in 1.0 au heliocentric orbit could detect $90 \%$ of EGs with $H \leq 22 \mathrm{mag}$ in $\sim 3.8 \mathrm{yr}$ of observing time. A more advanced constellation of nine $20 \mathrm{~cm}$ telescopes could detect $90 \%$ of $H=24.2 \mathrm{mag}$ (i.e., $\sim 50 \mathrm{~m}$ in size) EGs in less than $5 \mathrm{yr}$.
\end{abstract}

Key words. astrometry - techniques: image processing - minor planets, asteroids: general - space vehicles: instruments methods: data analysis - methods: observational

\section{Introduction}

The discovery and characterization of near-Earth objects (or NEO, which is an object that orbits the Sun and approaches or crosses the Earth's orbit) is motivated by several aspirations and concerns, including scientific research, planetary protection, and exploration efforts. NEOs are believed to be remnants from the early evolution of the solar system and hence studies of their dynamics and chemical composition may offer important information about conditions at that early epoch. The possibility that some NEOs could approach and even impact the Earth has motivated many observers worldwide to systematically search, catalog, and study the NEO population. Over the years, NASA has funded several NEO surveys to find such potentially hazardous objects (PHO).

To date, approximately 12300 NEOs have been discovered ${ }^{1}$, 820 of which have a diameter larger than $1 \mathrm{~km}$. From that population, 1492 objects $(12.2 \%)$ are classified as "potentially hazardous" with a minimum orbit intersection distance (MOID) of less than 0.05 astronomical units (au). A recent Report by the US National Research Council (NRC 2010) recognized the fact that objects smaller than $140 \mathrm{~m}$ in size are also capable of causing significant damage to the Earth. Estimates show (Harris $2009,2011)$ that there are millions of asteroids with sizes ranging from $140 \mathrm{~m}$ down to $30 \mathrm{~m}$ that are still undetected, but those objects are large enough to cause major regional damage in the event of an Earth impact. Early analysis of the object that entered the Earth's atmosphere over the Siberian wilderness near Podkamennaya Tunguska in 1908 estimated that its

\footnotetext{
1 The most recent information on the NEOs discovered may be obtained from the IAU's Minor Planet center at: http: //www . minorplanetcenter. net/
}

size was $\sim 70 \mathrm{~m}$. However, recent analyses (Chyba et al. 1993; Boslough \& Crawford 1997, 2008) indicate that the object could have been substantially smaller, perhaps 30 to $50 \mathrm{~m}$, causing much of the damage by exploding in the atmosphere and resulting in shock waves that devastated more than $2000 \mathrm{~km}^{2}$ of forest. Accordingly, NEOs as small as $30-50 \mathrm{~m}$ in size could be highly destructive. Among recent events, the Chelyabinsk meteor in 2013 had a diameter of only $17 \mathrm{~m}$ prior to entering the Earth's atmosphere. Therefore, in addition to the efforts of finding objects $140 \mathrm{~m}$ and larger, there is a need for detecting as many objects that are 30-50 m (and, perhaps, even smaller) as possible. The possibility of mining of near-Earth asteroids (NEAs, a subset of the NEOs) has also provided an impetus to private companies to perform a census of these objects to identify the most viable targets.

Recognizing the threat that NEOs pose to life on Earth, the US Congress has passed the 2005 NASA Authorization $\mathrm{Act}^{2}$, where NASA was mandated by the end of year 2020 to detect, track, catalog, and characterize at least $90 \%$ of NEOs brighter than $H=22$ mag that could potentially impact the Earth. (To guide the search for faint objects one uses either the size of a NEO or its brightness. However, because of the large variation in the bimodal distribution of albedos, there is no simple relationship between $H$ mag and size. In this paper, we have conducted simulations of a constellation of SmallSats to detect $90 \%$ of $H=22 \mathrm{mag}$ NEOs and in one case $H=24.2 \mathrm{mag}$ NEOs, which the $2010 \mathrm{NRC}$ report would refer to as $140 \mathrm{~m}$ NEOs and $50 \mathrm{~m}$ NEOs, correspondingly.) The US Congress also asked the NRC in 2008 to form a committee to determine the optimum

\footnotetext{
2 Specifically, the George E. Brown, Jr. Near-Earth Object Survey sec-
} tion of the NASA Authorization Act of 2005 (Public Law 109-155). 
approach to doing so. In 2010, after a detailed look at a number of alternative approaches, the NRC concluded that the goal of finding $90 \%$ of $H=22$ mag NEOs by the year 2020 was an almost impossible achievement (NRC 2010). The NRC committee looked at various ground- and space-based options and found that the most viable approaches with the potential to complete the survey in the period of approximately $10 \mathrm{yr}$ were the spacebased ones. Each of the corresponding missions would rely on a single spacecraft, would require an active decade-long observing campaign, and would cost over $\$ 500 \mathrm{M}$. However, even these expensive missions will not guarantee completion of the survey by 2020 .

The 2010 NRC report emphasized that a combination of the space-based efforts together with an appropriate ground-based facility (such as $\mathrm{LSST}^{3}$ or PanSTARRS ${ }^{4}$ ) could be used to accelerate the survey. Such a combination could complete the survey well before 2030, perhaps as early as 2022. They reported that using a large ground-based telescope alone (for instance, LSST), even if this facility would be fully dedicated to NEO search, could not complete the Congress-mandated survey by the original 2020 deadline. In fact, it would take a decade-long dedicated campaign to complete this effort probably just before 2030 . Furthermore, the committee concluded that, despite associated launch risk and a more limited lifetime, a space-based option could be the fastest means to complete the survey.

Since the release of the 2010 NRC report we witnessed significant technology progress in several relevant areas that could result in a major paradigm shift in the search for NEOs. One such area is the technique of synthetic tracking of NEOs (Shao et al. 2014), which makes it technically feasible to perform NEO searches with small CubeSat-compatible cameras. Synthetic tracking of NEOs is an emerging technology that was recently developed and demonstrated by NASA's Jet Propulsion Laboratory (JPL; Shao et al. 2014; Zhai et al. 2014). When used from a ground-based facility, this new tracking technique provides an order of magnitude improvement in the ability to detect and track dim and fast-moving objects. If implemented on a $10 \mathrm{~cm}$ CubeSat telescope in space with $800 \mathrm{~s}$ observations, synthetic tracking of NEOs would result in a sensitivity to a 20.5 mag object at signal-to-noise ratio $S / N=7$, making it an ideal candidate technology to use on a CubeSat.

Another technological advancement that enables the proposed small spacecraft mission architecture is the rapid development, flight heritage, and technology maturation for small and capable spacecraft, namely CubeSats. A single unit (1U) Cube$\mathrm{Sat}^{5}$ is a type of miniaturized satellite that usually has a volume of exactly one liter (based on a $(10 \mathrm{~cm} \times 10 \mathrm{~cm} \times 10 \mathrm{~cm}$ ) formfactor), has a mass of no more than $1.33 \mathrm{~kg}$ and typically costs $\$ 1-2 M$. In fact, we observe that a CubeSat-based mission architecture presents a viable alternative relative to the missions proposed in the NRC report. Modern CubeSats have benefited from decades of development efforts in miniaturization of many spacecraft technologies. Typically, modern CubeSats use commercial off-the-shelf (COTS) space-qualified hardware that can be purchased at a cost that is dramatically lower relative to that of conventional spacecraft components. The radically lower cost of a CubeSat-based architecture enables one to consider using multiple small spacecraft, each consisting of several CubeSat units,

\footnotetext{
The Large Synoptic Survey Telescope (LSST), for details, see http: //www. lsst.org/lsst/about

The Panoramic Survey Telescope and Rapid Response System (PanSTARRS), http://pan-starrs.ifa.hawaii.edu/public/

5 For information on CubeSats, please visit http://en.wikipedia. org/wiki/CubeSat and http://cubesat.jpl.nasa.gov/
}

while conventional multi-spacecraft architectures would never be economically viable. (A good example of an interplanetary CubeSat-based mission is the Mars Cube One (MarCO) mission, which is now ready for launch ${ }^{6}$. MarCO consists of two identical $6 \mathrm{U}$ CubeSats with the total cost for both being $\sim \$ 10.2 \mathrm{M}$. Interestingly, the actual incremental cost of the 2nd 6U CubeSat was $\$ 1.0 \mathrm{M}$. Thus, a constellation of six MarCOs with a total mass of $40 \mathrm{~kg}$ would cost $\sim \$ 15 \mathrm{M}$.)

We present a new mission concept that uses a constellation of SmallSats (that are in form-factor comparable with the 9U CubeSats) equipped with a $10 \mathrm{~cm}$ synthetic tracking telescope to survey over $90 \%$ of the population of $H=22$ mag Earth-grazing NEOs (with MOID of $\leq 0.02 \mathrm{au}$ ) in $\sim 3.8 \mathrm{yr}$ of observing time, all at a cost that is dramatically lower relative to any of the missions examined by the NRC. Furthermore, a second generation constellation of synthetic tracking SmallSats could survey $90 \%$ of $50 \mathrm{~m}$ NEOs in less than $5 \mathrm{yr}$.

This paper is organized as follows: in Sect. 2, we present the details of our recent simulation developed to study a search for $H=22$ mag NEOs using multiple SmallSats in heliocentric orbits whose form factor is comparable to $9 \mathrm{U}$ CubeSats. In Sect. 3, we discuss several SmallSats-based mission scenarios that could accomplish the Congress-mandated NEO search in $\sim 3.8 \mathrm{yr}$ of observing time. We also discuss a second generation SmallSats constellation that could search for $90 \%$ of NEOs with sizes down to $50 \mathrm{~m}$ in diameter. In Sect. 4, we summarize and discuss the results. Appendix A summarizes the synthetic tracking technique, which leads to high detection sensitivity when implemented with a $10 \mathrm{~cm}$ optics on a CubeSat. Appendix B discusses the emerging capabilities of interplanetary CubeSats.

\section{Simulation of a survey to find $90 \%$ of NEOs}

The 2010 NRC report examined combinations of a spacecraft and a dedicated ground-based NEO search facility (NRC 2010), although the idea of putting multiple spacecraft into a solar orbit was not examined, likely because of the prohibitively large cost of launching multiple $\sim 0.5-1.0$ billion dollar spacecraft. Furthermore, low-cost small spacecraft were not studied, likely because they were perceived as incapable of accomplishing this mission.

Compared to the approaches in the NRC report, our strategy towards accomplishing the NEO search is different: in particular, as opposed to using a single spacecraft, we explored the advantages offered by constellations of multiple spacecraft relying on small telescopes and a synthetic tracking technique (see Appendix A) for this purpose. To examine the advantages of using multiple spacecraft, we developed a simulator that takes into account the current model for distribution of the NEOs, expected performance of the synthetic tracking camera on a CubeSat, and the $9 \mathrm{U}$ NEO CubeSat operational characteristics, as well as contributions from the anticipated zodiacal dust background, and so on.

We simulated a number of NEO observing scenarios using multiple spacecraft in solar orbits. The methodology of our simulation was designed to mimic the survey simulations discussed in the NRC 2010 report, with the exception of using mission architectures consisting of multiple spacecraft.

We used the published debiased orbital and absolute magnitude distribution of NEOs (Granvik et al. 2016) that accounts for correlations in various orbital elements of NEOs. In the $\mathrm{NEO}$ population we took the NEO orbital parameters from

\footnotetext{
6 See http://www.jpl.nasa.gov/cubesat/missions/marco. php
} 

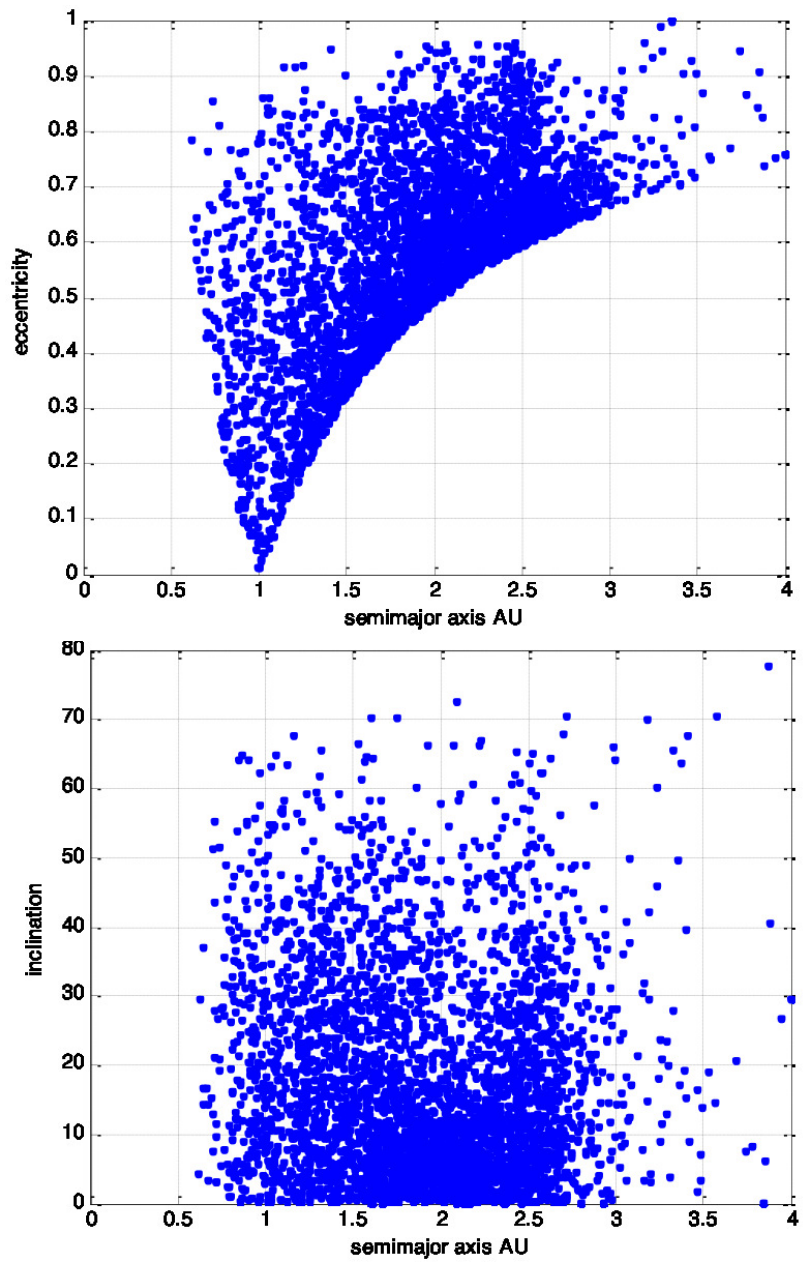

Fig. 1. Orbital distribution of $\sim 10000$ "impactors" found within the parent distribution taken from (Granvik et al. 2016).

(Granvik et al. 2016) for semi-major axis, eccentricity and inclination, but randomized the time of periastron. The Granvik population calculates the MOID, and for the Earth grazers, we chose only the NEOs with MOID $<0.01$ au but chosen so that there were approximately 8000-12000 objects. These objects are the ones most likely to impact the Earth at some time in the future, we call them Earth grazers (EG). The distribution of semi-major axis, eccentricity, and inclination for EGs are shown in Fig. 1.

The SmallSat constellations were calculated using the following approach: although each of the $N$ spacecraft could have an arbitrary orbit, we first examined those constellations where all the spacecraft had the same semi-major axis and inclination, but were uniformly distributed around the Sun. The simulation then stepped through $N$ to simulate constellations with variable numbers of spacecraft, typically considering scenarios up to $20 \mathrm{yr}$. The time step for the simulation was $\sim 810 \mathrm{~s}$ (representing $800 \mathrm{~s}$ integration time at one field of view (FOV) and $10 \mathrm{~s}$ slew to next field). The time step was selected to represent the combination of the integration time and the time needed to slew the telescope to an adjacent FOV.

At each time step, the telescope would point at a particular part of the sky. Every EG was checked to determine if and when it was in the camera's FOV. If it was, the apparent magnitude of the EG was calculated, given its $H$ magnitude, distance from the Sun and distance from the CubeSat and the phase angle (the angle between the telescope and the Sun as seen from the EG). The telescopes scanned the sky in an "orange peel" pattern and always satisfied the $65^{\circ}$ Sun-avoidance constraint. Currently, we do not simulate the loss of sensitivity when the EG image would be streaked, because it is not relevant with synthetic tracking.

For a search of $H=22$ mag EGs we look at a $10 \mathrm{~cm}$ aperture camera with a limit magnitude of 20.49 mag similar to many existing and near-future ground-based observatories $\left(\mathrm{CSS}^{7}\right.$ and ATLAS, $\mathrm{PTF}^{8}$ ) not as sensitive as EG surveys with larger telescopes (i.e., PanSTARRS, LSST). This camera is also less sensitive than all of the space missions mentioned in the NRC report. With a $4 \mathrm{~K}$ sensor and $3.3^{\prime \prime}$ pixels the FOV $\sim 14\left({ }^{\circ}\right)^{2}$ is similar to the IR space telescopes in (NRC 2010).

\section{Case studies}

We first validated our modeling approach and assumptions by reproducing the results for the IR telescope in Venus-trailing orbit from the 2010 NRC report (i.e., Sect. 5.1 in (NRC 2010)). We then investigated the trade-offs between telescope FOV, sky coverage rates, and survey time to verify the choice of the proposed aperture design for the CubeSat and scanning strategy. We also explored different constellation sizes and heliocentric orbits to identify optimal constellation parameters that could determine the optimal trade-off between survey size. Finally, we investigate the ability of a constellation of SmallSats that relies on the CubeSat-derived technology to detect $90 \%$ of smaller EGs with sizes down to $50 \mathrm{~m}$ in diameter. Below we present all these special cases and discuss results obtained.

\subsection{Case 1: comparison to the proposed IR telescope on a Venus-like orbit}

To verify our simulation approach, we first aim to replicate the results for the NRC-proposed IR telescope in a Venus-like orbit. Our objective was to verify that our simulation was consistent with those conducted for the 2010 NRC study. The information for this mission was obtained from publicly available resources ${ }^{9}$ and email correspondence (H. Reitsema 2014, priv. comm.). The IR telescope on a Venus-like orbit is sufficiently sensitive to detect a $140 \mathrm{~m}$ EG from a distance of $0.6 \mathrm{au}$. For a telescope at a distance of 0.7 au from the Sun, such a sensitivity yields a limiting magnitude of 21.5 mag or $\sim 1$ mag more sensitivity than the telescope on our proposed CubeSat mission. The IR telescope would integrate for a total of $180 \mathrm{~s}$ over six $30 \mathrm{~s}$ exposures followed by a $60 \mathrm{~s}$ slew/settle period. These six exposures will be used to remove cosmic ray events before co-adding the frames.

The scanning strategy consists of revisiting areas of the sky $\sim 1 \mathrm{~h}$ apart to confirm the detection of moving objects. We implemented an orange peel scanning pattern where the FOV of the CubeSat camera was properly taken into account. Assuming the FOV of $11\left({ }^{\circ}\right)^{2}$ (similar to that of the Sentinel mission ${ }^{10}$ ), we modeled each observation as two observations of $240 \mathrm{~s}$ including slew times separated by $1 \mathrm{~h}$. When observing an EG that is at phase angle of $90^{\circ}$ from the Sun, only half of the surface is in sunlight. In the visible band, the apparent brightness of an

\footnotetext{
7 The Catalina Sky Survey (CSS), http: //www . lpl . arizona . edu/ css/

8 The Palomar Transient Factory, http://www.ptf.caltech.edu/ iptf

9 See information on the Sentinel mission: http:// sentinelmission.org/

${ }^{10}$ http://sentinelmission.org/sentinel-mission/ sentinel-data-sheet/
} 


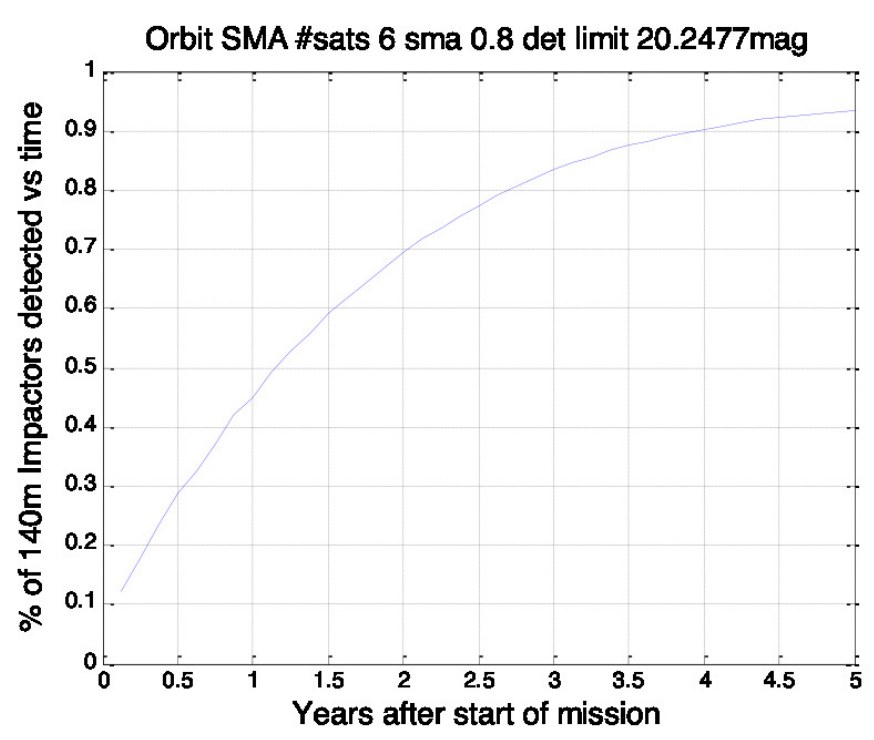

Fig. 2. Validation of our model by reproduction of Venus IR results (NRC 2010). The spacecraft is on a circular orbit at 0.7 au from the Sun. The camera's FOV $=11\left({ }^{\circ}\right)^{2}, 420 \mathrm{~s}$ integration time, $60 \mathrm{~s}$ slew time (covering $\left.1980\left({ }^{\circ}\right)^{2} / 24 \mathrm{~h}\right)$; threshold magnitude $=21.5$.

EG at $90^{\circ}$ phase angle is approximately one third of its brightness at opposition. This phase angle effect was turned off in our simulation of the IR telescope.

We found that using our simulation approach, the Venus IR mission required $\sim 7.8 \mathrm{yr}$ to find $90 \%$ of $H=22$ mag EGs as in Fig. 2, which is within 10-20\% of the $7.5 \mathrm{yr}$ in (NRC 2010). A small discrepancy is expected because there may be differences in the modeling assumptions relative to (NRC 2010). However, overall, these results confirm our major assumptions and provide confidence that our general simulation approach is correct.

\subsection{Case 2: survey time versus sky cover/camera FOV}

We next studied the importance of the synthetic tracking camera FOV size in its effectiveness to detect $90 \%$ of 140 m EGs. The goal is to identify the optimal FOV size and to understand where our baseline CubeSat scanning strategy with $\sim 1500\left({ }^{\circ}\right)^{2} /$ day is relative to these trades.

Unlike conventional CCD-based exposures (which cannot trade integration time and sensitivity), with synthetic tracking we have the flexibility to trade the limiting magnitude for the sky coverage. Doubling the integration time results in the $\mathrm{S} / \mathrm{N}$ improving by $2^{1 / 2}$, the detection distance increasing by $2^{1 / 4}$, and the volume of space covered increasing by $2^{3 / 4}$; the sky coverage rate $\left(\right.$ in $\left({ }^{\circ}\right)^{2} /$ day), however, drops by a factor of 2 . With only one telescope, shorter integration times are preferred because they enable us to cover the sky more quickly, despite the reduction in $\mathrm{S} / \mathrm{N}$ and sky volume coverage. It takes a finite amount of time to slew and settle the telescope, so that the telescope may capture the next area of sky. For a given slew time, it is relatively easy to calculate the optimum exposure time, which is approximaterly three times the slew time when the goal is to maximize the number of objects viewed per hour. However, when searching for EGs, maximizing the coverage per hour is not an appropriate objective. This is because the population of available objects changes slowly, on timescales of $\sim 2$ months. Therefore, there is a trade-off between the sensitivity (which improves with a smaller sky FOV) and the frequency of scanning the sky (which improves with a larger sky FOV).

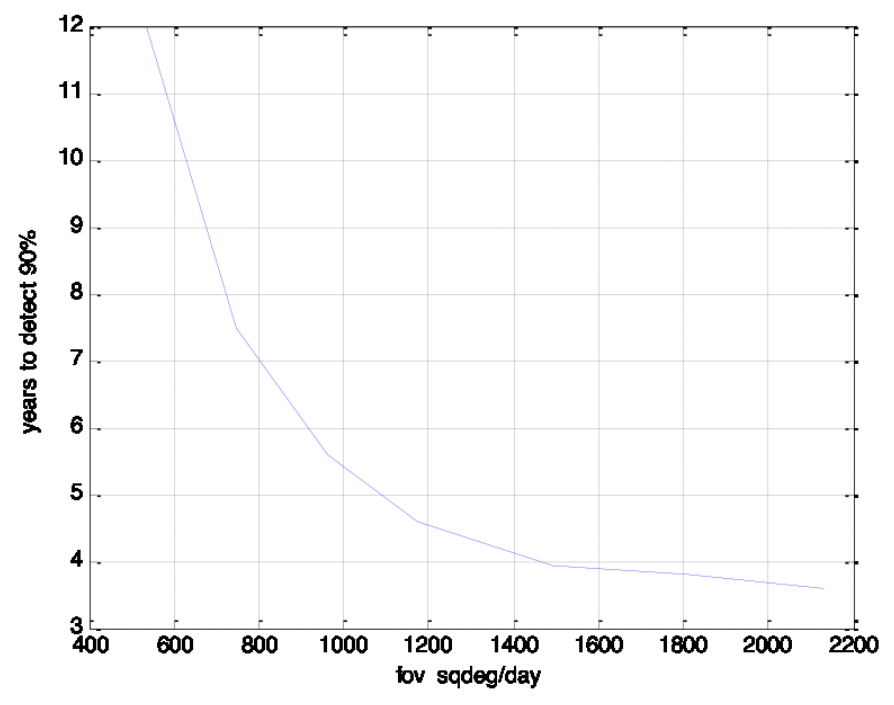

Fig. 3. Time to find $90 \%$ of $H=22$ mag EGs detected as a function of FOV size and daily sky coverage for ideal constellation with six CubeSats at 0.85 au with variable FOVs, $800 \mathrm{~s}$ integration, $10 \mathrm{~s}$ slews and 20.49 mag limit when the telescope was at 1 au.

To evaluate the impact of the FOV on performance, we chose the constellation with six CubeSats at 0.85 au and simulated a range of FOVs between 0.25 and $25.0\left({ }^{\circ}\right)^{2}$ (i.e., 600-2400 $\left({ }^{\circ}\right)^{2}$ in $24 \mathrm{~h}$ ), as shown in Fig. 3 (assuming all had a 20.49 mag limit at $1 \mathrm{au})$. We note that for FOVs $<3\left({ }^{\circ}\right)^{2}$ the time to complete the survey exceeded $20 \mathrm{yr}$, but the exact time was not solved; thus, these results are not shown in Fig. 3. The point where there are diminishing returns with increasing the FOV (or the knee in these plots) is around $14\left({ }^{\circ}\right)^{2}$ (i.e., $1500\left(^{\circ}\right)^{2} /$ day), where for greater FOVs, the time to detect $90 \%$ of EGs was not affected significantly, but for smaller FOVs the detection time grows exponentially. Figure 3 depicts the effectiveness of an EG search campaign as a function of the FOV of a telescope. It shows that for a search for $90 \%$ of EGs with $H \leq 22$ with a facility that has a limiting magnitude of $\sim 20.5$ mag, a scanning rate of more than $\sim 1500\left({ }^{\circ}\right)^{2} / 24 \mathrm{~h}$ is an inefficient use of resources. The PanSTARRs telescope operating $10 \mathrm{~h}$ per night would survey $1400\left({ }^{\circ}\right)^{2}$ every observing night. From these results we can conclude that increasing the sky coverage from $1400\left(^{\circ}\right)^{2}$ to $20000\left({ }^{\circ}\right)^{2}$ per night with more ground-based observatories would not significantly reduce the time to find $90 \%$ of $140 \mathrm{~m}$ EGs. A space mission in an Earth orbit duplicates the search volume of the ground-based observatories.

\subsection{Case 3: design of interplanetary CubeSat constellation}

Next, we investigate CubeSat constellation architectures to accomplish the mission. The simulation environment described above was used to study different numbers of spacecraft in the constellation at various heliocentric ranges. For the multispacecraft architectures, we assumed that the spacecraft were equally distributed in solar orbits. The telescope magnitude limit was assumed to be 20.49 mag at 1 au, and zodi effects considered with FOV $=14\left({ }^{\circ}\right)^{2}$ (see Appendix B). The telescope is assumed to integrate for $800 \mathrm{~s}$ and then the spacecraft slews for $10 \mathrm{~s}$ (to model the analogous approach of a $400 \mathrm{~s}$ observation and $5 \mathrm{~s}$ slew time repeated twice between different pointings, which covers $\sim 1500\left(^{\circ}\right)^{2} / 24 \mathrm{~h}$. 


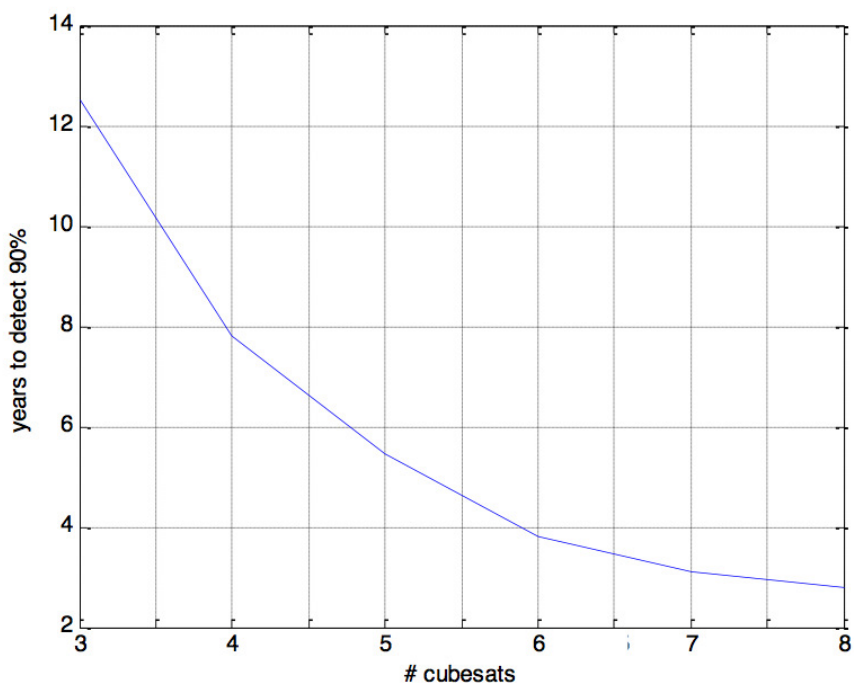

Fig. 4. Time to detect $90 \%$ of $H=22$ mag EGs assuming $\mathrm{FOV}=14\left(^{\circ}\right)^{2}$, $800 \mathrm{~s}$ integration, $10 \mathrm{~s}$ slews, covering $\sim 1500\left({ }^{\circ}\right)^{2}$ per $24 \mathrm{~h}$, with slewing model "on". The figure shows sensitivity to the number of CubeSats for constellations 0.85 au from the Sun. In both cases, the CubeSats are distributed equally around the Sun.

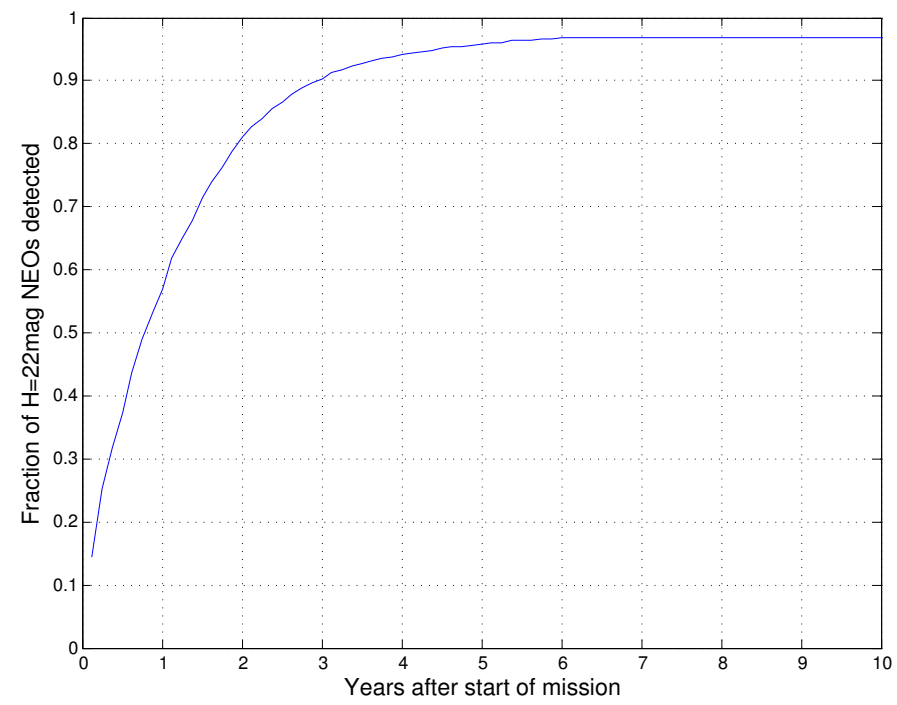

Fig. 5. Fraction of $H=22 \mathrm{mag}$ EGs detected for constellations of CubeSats from Fig. 4 with FOV $=14\left({ }^{\circ}\right)^{2}, 800 \mathrm{~s}$ integration, $10 \mathrm{~s}$ slews, covering $\sim 1500\left({ }^{\circ}\right)^{2} / 24 \mathrm{~h}$, with slewing model active.

We performed simulations for different constellation sizes at various heliocentric ranges from 0.7 to $1.1 \mathrm{au}$. The results showing the time to detect $90 \%$ of $H=22$ mag EGs for an idealized constellation are in Fig. 4. There is a broad range of semi-major axis between 0.85 and 1.0 au where the time to find $90 \%$ of EGs is minimized. The minimum occurs due to three competing effects. As the CubeSat constellation gets closer to the Sun, the sky background increases which leads to reduced sensitivity and increased survey times. On the other hand, a larger and larger percentage of the EGs will be detected at a large phase angle, again reducing sensitivity when semi-major axis exceeds $\sim 1$ au. Choice of smaller semi-major axis allows to minimize detection time because the orbits have shorter periods, covering the sky faster, and orbits that are closer to the perigees of the EGs. These
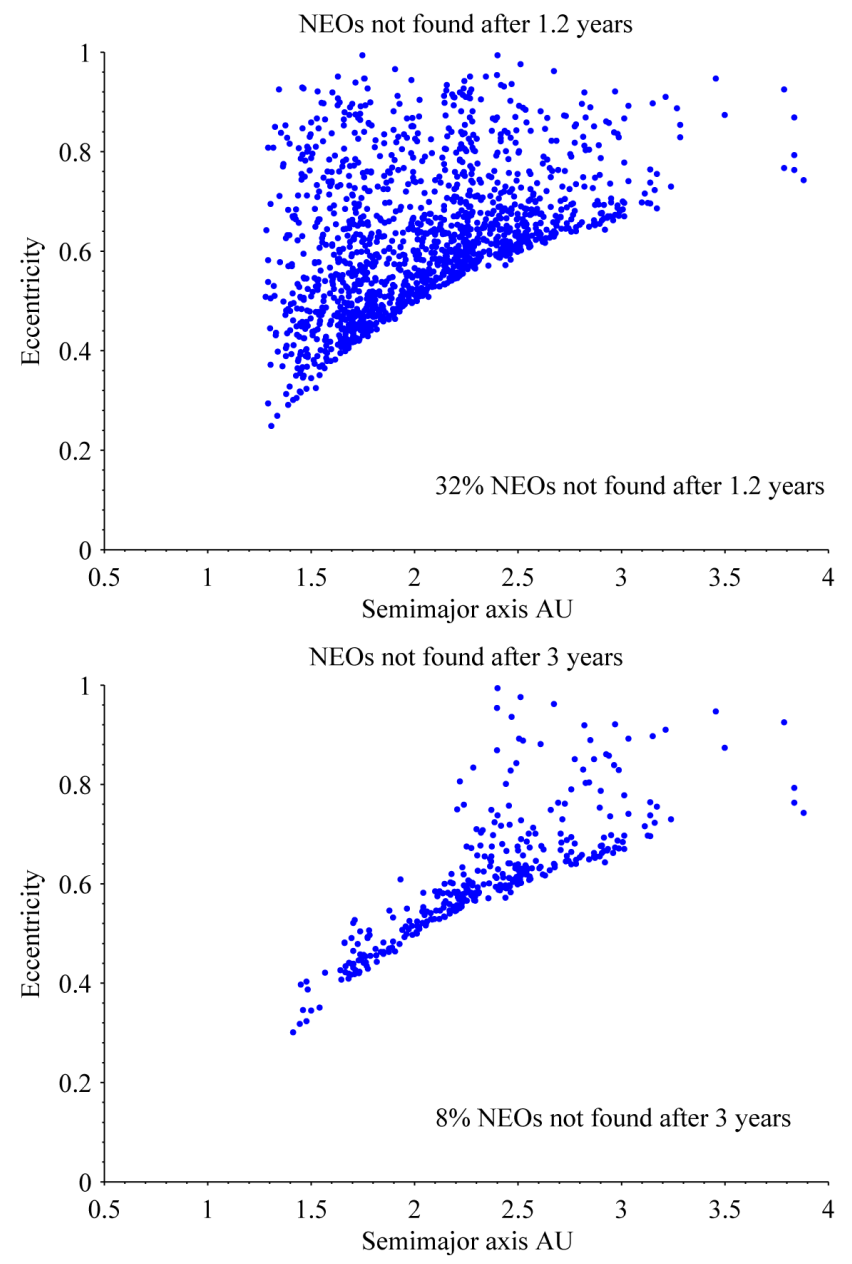

Fig. 6. Orbital properties of EGs that were not found after $1.2 \mathrm{yr}$ (left) and $3.0 \mathrm{yr}$ (right) with representative six-CubeSat constellations.

competing effects produce the broad minimum between $0.85 \mathrm{au}$ and $1.0 \mathrm{au}$. Looking at Fig. 4, we observe that addition of a new CubeSat to the constellation of $N$ spacecraft generally reduces the total time needed to complete the survey, where this reduction is most significant for small numbers of CubeSats. However, with each additional spacecraft, the additive search performance gain is reduced, with very little gain after $N \geq 6$. Figure 5 shows the time to find $90 \%$ of $H=22$ mag EGs with a constellation of six CubeSats placed on orbits with a semi-major axis of $\sim 0.85$ au.

We also note that the mission time never falls below $2 \mathrm{yr}$, even for constellations with up to $N=10$. This is due to the fact that with six uniformly-distributed spacecraft in solar orbit it takes nearly 2 yr to find almost $90 \%$ of all the EGs with magnitude less than $H=22$. The remaining "undiscovered" EGs have semi-major axis ranging from 2-3 au, spending most of their orbits beyond the accessibility of the CubeSats placed on solar orbit with semi-major axis of 0.85-1.0 au, as in Fig. 6. Capturing these EGs is simply a waiting game until they approach their periapsis and are close enough to a spacecraft to be detected.

\subsection{Case 4: detection of smaller EGs}

The 2010 NRC report concluded that a survey to find $90 \%$ of $140 \mathrm{~m}$ EGs would require a space mission with a lifetime of 8-10 yr. In addition to surveying for large EGs, the report also 


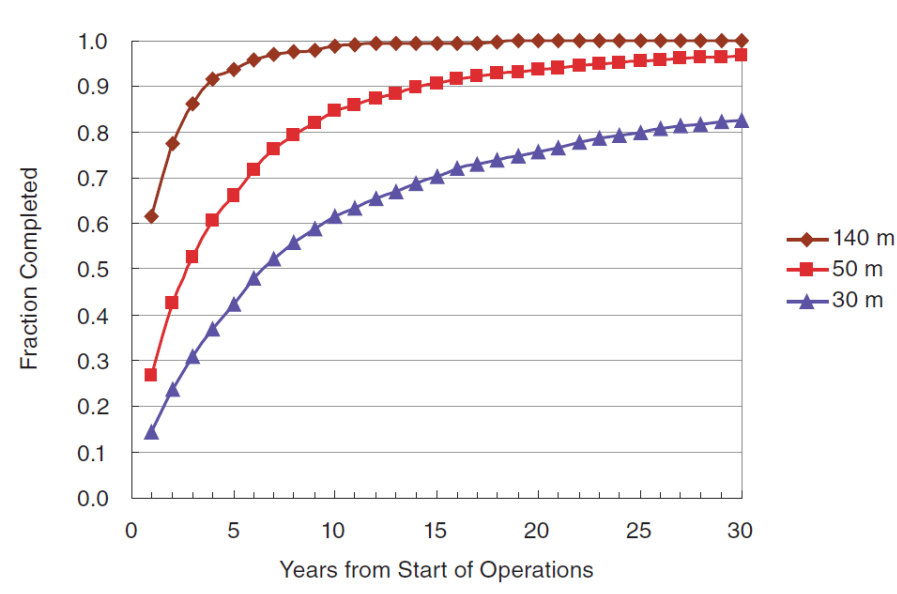

Fig. 7. Years to completion for $0.5 \mathrm{~m}$ IR telescope in a Venus-like orbit and a dedicated LSST (Fig. 3.10 from (NRC 2010)).

emphasized the need to look for smaller EGs, with sizes down to $30 \mathrm{~m}$, for planetary protection purposes. Figure 7 is from the NRC report showing the effectiveness of an EG search campaign with a combined IR telescope in a Venus-like orbit and a dedicated LSST. If completely dedicated to EG search, LSST would be a very powerful facility. If we ignore the loss of sensitivity from streaked images, LSST would be able to detect 25 mag objects. However, the combination of these two facilities represents a relatively large investment, and even then, detection of $90 \%$ of 50 m EGs would take $\sim 14$ yr. The NRC report ended by saying that detection of these smaller EGs is extremely challenging and expensive. We note that a typical operating cost of a major observatory is $\sim 10 \%$ of it's construction cost per year. In the case of LSST, whose total construction cost is estimated to be $\sim \$ 700 \mathrm{M}^{11}$, operating it even for a few years while $100 \%$ devoted to NEO search would be much more expensive than a constellation of Cubesats.

We re-examined this conclusion with a constellation of CubeSats with synthetic tracking cameras. Specifically, given the higher sensitivity enabled by synthetic tracking and high sky coverage rates at affordable costs provided by CubeSat platforms, we were interested in the potential for the proposed architecture to detect $90 \%$ of $50 \mathrm{~m} \mathrm{EGs} \mathrm{in} \mathrm{less} \mathrm{than} 10 \mathrm{yr}$.

The $50 \mathrm{~m}$ EGs are almost a factor of 10 dimmer than the $140 \mathrm{~m}$ EGs, with $H=24.2 \mathrm{mag}$. If the observatory is at $0.7 \mathrm{au}$, a $140 \mathrm{~m} \mathrm{EG}(H=22 \mathrm{mag})$ could be detected at ranges up to $0.4 \mathrm{au}$ away, if the detection magnitude limit was 20.5 mag. However, an EG with $H=24.2 \mathrm{mag}$ ( $\sim 50 \mathrm{~m}$ in size) would have to be at a range of no greater than 0.20 au from the telescope and at opposition to be detected. At any one time, the volume of the search space is eight times smaller. The search for these smaller EGs would require a more capable constellation than the strawman constellation we described for $H=22$ mag EGs. We also make some assumptions as to what type of detectors and small telescopes would be available in the near future. We pick one constellation as an example to illustrate the power of this approach realizing that optimizing the constellation design requires more work.

We assume that $(8 \mathrm{~K} \times 8 \mathrm{~K})$ detectors will become available, with other performance parameters similar to current smallerformat sCMOS detectors. The assumptions for this much more capable camera/constellation are listed in the Table 1. This

\footnotetext{
11 To that extent, see the relevant discussion of the costs involved at https://www.nsf.gov/news/news_summ.jsp?cntn_id=124899
}

Table 1. Parameters for an advanced synthetic tracking camera.

\begin{tabular}{rcc}
\hline \hline Parameters & Value & Units \\
\hline Telescope Diameter & 20 & $\mathrm{~cm}$ \\
Image & $2 \mathrm{X}$ & diff limit \\
Pixel size & 1.6 & arcsec \\
Effective background & 2.8 & arcsec \\
Magnitude limit & 22.15 & $\mathrm{mag}$ \\
Integration time & 800 & $\mathrm{~s}$ \\
Number of satellites & $\mathbf{9}$ & \\
EG size & 50 & $\mathrm{~m}$ \\
H magnitude & 24.24 & $\mathrm{mag}$ \\
\hline
\end{tabular}

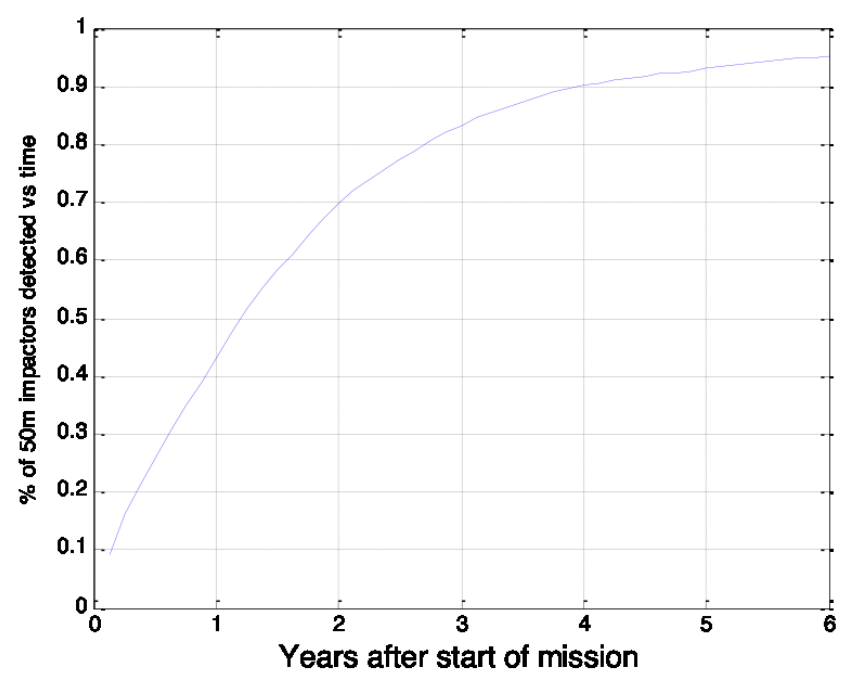

Fig. 8. Time to detect $90 \%$ of $50 \mathrm{~m}$ EGs with the constellation of SmallSats from Table 1.

constellation would detect $90 \%$ of $50 \mathrm{~m}$ EGs $(H=24.2 \mathrm{mag})$ more than once in approximately $4.5 \mathrm{yr}$, and compares favorably to all the concepts examined in the 2010 NRC report.

In solar orbits at $0.85 \mathrm{au}$, and a solar avoidance angle of $65^{\circ}$, a constellation of nineCubeSats with $20 \mathrm{~cm}$ apertures will detect $90 \%$ of 50 m EGs in $\sim 40 \mathrm{yr}$, as shown in Fig. 8.

This example illustrates the potential for a constellation of CubeSats to conduct a $90 \%$-complete search for $50 \mathrm{~m}$ EG that, according to the NRC report, would otherwise take a long time at a prohibitively high cost. These examples are not yet optimized to identify the configuration with minimal detection time or cost. Nevertheless, they show synthetic tracking with CubeSats may be the only affordable architecture to search for EGs significantly smaller than $140 \mathrm{~m}$.

The results above demonstrate the advantages of the proposed approach based on a constellation of CubeSats with synthetic tracking to search for EGs with various sizes. Such a constellation could conduct a $90 \%$ complete survey in significantly less time and lower cost relative to what was indicated in the NRC report. A more detailed and thorough examination of CubeSat constellations aiming at detecting $90 \%$ of $\sim 35-100 \mathrm{~m}$ EGs will be the topic of a future paper.

\section{Discussion and summary}

The time required to detect $90 \%$ of EGs with $H \leq 22$ mag depends on a number of factors, including limiting magnitude 
sensitivity, size of the FOV, and number and configuration of spacecraft. Our $10 \mathrm{~cm}$ CubeSat-based camera and ground-based telescopes such as $\mathrm{ZTF}^{12}$, CSS, and ATLAS have a limiting magnitude of $\sim 20.5 \mathrm{mag}$. Larger telescopes, such as PanSTARRs and LSST with smaller pixels ("/pixel) are more sensitive. Spacebased $50 \mathrm{~cm}$ IR telescopes are also more sensitive ( $21.5 \mathrm{mag})$ relative to similar ground-based visible cameras. Simulations conducted for (NRC 2010) showed the importance of the distribution of the observatories. For ground-based observatories, a distribution in geographic latitude helps with sky coverage. The NRC report also hinted at the importance of distributing observatories around the solar system. The most capable combination of observatories examined in (NRC 2010) was LSST and an IR telescope in a Venus orbit. A Venus-like orbit is advantageous relative to one on Earth or in an Earth orbit because this type of telescope would essentially duplicate much of what LSST would detect.

Our survey simulations in Sect. 3.2 show that sky coverage beyond $\sim 1400\left({ }^{\circ}\right)^{2}$ per $24 \mathrm{~h}$ would not significantly decrease the time needed to find $90 \%$ of $H=22$ mag EGs. It should be noted that PanSTARRs with its $7\left({ }^{\circ}\right)^{2}$ FOV and spending $4 \times(30 \mathrm{~s}+15 \mathrm{~s})$ per FOV would cover $\sim 1400\left({ }^{\circ}\right)^{2}$ in $10 \mathrm{~h}$. Adding more ground-based telescopes without increasing sensitivity would not shorten the time to conduct a $90 \%$ complete survey.

Earlier in the introduction we mentioned that reaching high astrometric precision in measuring the orbital parameters of EGs is as important as detecting them. For planetary protection purposes, the goal of finding $90 \%$ of $H=22$ mag EGs is not sufficient if we do not have the data to determine weather or not a newly discovered asteroid will impact the Earth or not. If the astrometric accuracy is $0.2^{\prime \prime}$, and the EG is observed a half dozen times on its first pass, for the observational data arc of 13 days the orbit derived from those measurements is rather poor. In fact, it is so poor that if the EG were to revisit $\sim 4-5$ yr later, we would not know where to point the telescope within $\approx 10^{\circ}$.

Currently a high accuracy orbit will require one of two circumstances: one is where several observations during one apparition are augmented by radar observations. The other is where observations at multiple apparitions are possible. Our simulations of a nominal six-SmallSat constellation show that, on average, each EG is detected approximately ten times by several different SmallSats over a $\sim 6$ yr period. Our simulations also showed that $\sim 78 \%$ of the EGs would be detected 4 or more epochs over $a>120^{\circ}$ orbital arc. These $\sim 4+$ observations, each with $\sim 0.5^{\prime \prime}$ astrometry per observation, may be sufficient to measure the EG orbit to measure the MOID with Earth's orbit to a few times the Earth's diameter. A more detailed examination of this issue will be addressed elsewhere.

Here we look at a constellation of SmallSats in solar orbit capable of detecting $90 \%$ of EGs one or several times during the entire observing campaign. We compared results for various SmallSat constellations to the results of the 2010 NRC report. We note, that if only a single detection of an asteroid is made, that is far from what is needed to determine if that asteroid would eventually hit the Earth. Ideally, a survey would not only detect the objects, but detect them enough times to measure an accurate orbit. If accurate orbit measurements of a significant fraction of EGs is not possible, then it is preferable to have enough measurements of EGs that those measurements can be linked with

\footnotetext{
12 The Zwicky Transient Facility (ZTF) is a new time-domain survey that will have first light at Palomar Observatory in 2017. For details, please visit: http://www.ptf. caltech.edu/ztf
}

the measurements of another survey. These topics are beyond the scope of this paper and will be addressed in a subsequent paper currently in preparation.

We briefly address the topic of linking observations from a constellation of SmallSats. To study this problem, in one of the simulations, we adopted a different scan pattern, one designed to enhance "linked" observations. Instead of spending $800 \mathrm{~s}$ then moving to an adjacent FOV, we split the $800 \mathrm{~s}$ into two $400 \mathrm{~s}$ blocks $2 \mathrm{~h}$ apart. The entire $800 \mathrm{~s}$ was treated as a single data cube, as discussed in Appendix B.2. This resulted in a velocity measurement that was $\sim 0.5^{\prime \prime} /(2 \mathrm{~h})$. That same area was again scanned 2 days later again with $800 \mathrm{~s}$ total integration time. This cadence would be repeated $\sim 25$ days later when the whole sky was rescanned. The 2 nd epoch has to be $\sim 25$ days later in order for the two sets of measurements to be connected with low false-positive and low false-negative connection. The original scan pattern $810 \mathrm{~s}$ for a $14.1\left(^{\circ}\right)^{2} \mathrm{FOV}$, would scan $\sim 3 \pi$ steradian every $\sim 20$ days. This doubled scan pattern would have a natural "repeat" cycle time of $\sim 40$ days. In order to reduce this time, the "linked" scan pattern had a slightly larger Sun-avoidance angle, but more importantly it avoids scanning the ecliptic poles $\left( \pm 45^{\circ}\right.$ ecliptic latitude).

This nominal constellation of six SmallSats would provide linked observations of $79 \%$ of $H=22$ mag EGs in six years and $95 \%$ of EGs would be detected at least once. This cadence of observations, called a "bottom up" approach, enables the linking observations of the same object observed at different times by different satellites. The other approach is a "top down" approach. With astrometric accuracy $<1^{\prime \prime}$, observation of the same asteroid four times when the orbital arc is $\gtrsim 120^{\circ}$ results in a very accurate orbit. If three of the observations were of one asteroid and the 4th of a 2 nd asteroid, the fit residuals would be large.

The low cost of an interplanetary CubeSat presents the opportunity of a mission architecture consisting of launching multiple spacecraft in solar orbit. With low anticipated cost, this option is not only feasible, it is also highly attractive. In fact, a mission relying on multiple spacecraft allows for added mission redundancy, effective sky coverage, and a shorter period to complete the EG search and provides chances for frequent technology upgrades. With preliminary cost estimates for interplanetary SmallSats being so low, a further cost reduction would come from the fact that additional CubeSats would cost only a fraction of the original cost $(50 \%$ or less due to recurring engineering costs). Therefore, the cost of launching six to ten interplanetary SmallSats is still expected to be an order of magnitude lower than that for the missions in the 2010 NRC report.

Significant improvements are expected from optimizing the constellation design, scanning strategies, FOVs, and anticipated SmallSat lifetimes. A rigorous analysis of the optimal number of spacecraft to mitigate risks and ensure a high probability of mission success in the desired time for an acceptable cost is beyond the scope of this paper; it will be presented elsewhere.

The mass-produced space-qualified hardware used in small satellites dramatically reduces the cost of a space observatory making a constellation of these telescopes not only affordable, but also significantly lower in cost relative to conventional medium-sized space telescopes such as those in the NRC report. Furthermore, EGs much smaller than $H \leq 22$ mag (or $\sim 140 \mathrm{~m}$ in size) can still cause major damage when they impact the Earth. We emphasize that the only affordable way to survey $90 \%$ of $70 \mathrm{~m}$ or $50 \mathrm{~m}$ EGs would be with the synthetic tracking multiple SmallSat architecture. We will investigate the relevant mission design and architecture in a subsequent publication. 
In conclusion, we observe that by combining synthetic tracking and CubeSat technologies, compared to all survey architectures and methods proposed previously, we are fundamentally "playing in a different ball park". This new paradigm is both much less expensive and significantly more capable of finding not just $140 \mathrm{~m}$ EGs in much less time, but also $90 \%$ of smaller EGs with sizes down to $50 \mathrm{~m}$. We note that even a factor of two increase in our cost estimates would still be a fraction of the cost of the missions/facilities mentioned in NRC (2010). Clearly, more mission design work is needed. Therefore, the potential of a constellation-based architecture presented here will be explored further.

Acknowledgements. We thank Paul Chodas, Steve Chesley, T. Joseph W. Lazio and Robert Preston of JPL for their interest and many useful discussions during the work and preparation of this manuscript. It is our pleasure to thank Prof. Edward (Ned) L. Write of UCLA for valuable comments and suggestions. We also thank Mikael Granvik for valuable comments on the manuscript and also for allowing us to use the NEO distribution model from (Granvik et al. 2016). The work described here was carried out at the Jet Propulsion Laboratory, California Institute of Technology, under a contract with the National Aeronautics and Space Administration.

\section{References}

Bernstein, G. M., Trilling, D. E., Allen, R. L., et al. 2004, AJ, 128, 1364 Boslough, M. B. E., \& Crawford, D. A. 1997, Ann. NY Acad. Sci., 822, 236 Boslough, M. B. E., \& Crawford, D. A. 2008, Int. J. Impact Eng., 35, 1441 Chyba, C. F., Thomas, P. J., \& Zahnle, K. J. 1993, Nature, 361, 40
Duncan, C. B., Smith, A. E., \& Aguirre, F. H. 2014, in Proc. AIAA/USU Conference on Small Satellites, paper: SSC14-IX-3, http://digitalcommons.usu.edu/smallsat/2014/AdvTechComm/3, Logan, Utah, USA

Fazio, M., MacNeal, K., \& Sheldon, D. 2014, in Electronics Technology Workshop, NASA Electronic Parts and Packaging Program (NEPP)

Granvik, M., \& Muinonen, K. 2005, Icarus, 179, 109

Granvik, M., \& Muinonen, K. 2008, Icarus, 198, 130

Granvik, M., Muinonen, K., Jones, L., et al. 2007, Icarus, 192, 475

Granvik, M., Morbidelli, A., Jedicke, R., et al. 2016, Nature, 530, 303

Gural, P. S., Larsen, J. A., \& Gleason, A. E. 2005, AJ, 130, 1951

Harris, A. W. 2009, The NEO population, impact risk, progress of current surveys, and prospects for future surveys, presentation to the Survey, Detection panel of the nrc committee to review near-earth object surveys and hazard mitigation strategies, Jan. 28-30, 2009, Space Science Institute

Harris, A. W. 2011, The NEO population, presentation at the target neo workshop, george washington university, feb. 22, 2011, http://targetneo. jhuapl.edu/pdfs/sessions/targetneo-session2-harris.pdf, Space Science Institute, Consultant to NASA/JPL NEO Program Office

Leinert, C., Richter, I., Pitz, E., \& Hanner, M. 1982, A\&A, 110, 355

Marrese-Reading, C. M., Ziemer, J. K., Scharf, D. P., et al. 2010, in Proc. IEEE Aerospace Conference, Big Sky, MT

Mueller, J., Hofer, R., \& Ziemer, J. 2010, in Proc. 57th Joint Army-Navy-NASAAir Force (JANNAF) Propulsion Meeting, Colorado Springs, CO

NRC 2010, Defending Planet Earth: Near-Earth Object Surveys and Hazard Mitigation Strategies, Tech. Rep., National Research Council

Roach, F. E., \& Gordon, J. L. 1973, The Light of the Night Sky, Geophysics and Astrophysics Monographs, Book 4; Springer, December 1, 1973

Shao, M., Nemati, B., Zhai, C., et al. 2014, ApJ, 782, 1

Spangelo, S., Dalle, D., \& Longmier, B. 2015, AIAA/AAS Astrodynamics Specialist Conference (San Diego, CA, 2015), http://dx.doi.org/10. 2514/6.2014-4125

Tanga, P., \& Mignard, F. 2012, Planetary and Space Science, 73

Zarifian, P., et al. 2014, Asteroid Detection with CubeSats using synthetic tracking, JPL TeamXc report \#1555 on the study 2014-12, JPL

Zhai, C., Shao, M., Nemati, B., et al. 2014, ApJ, 792, 60 


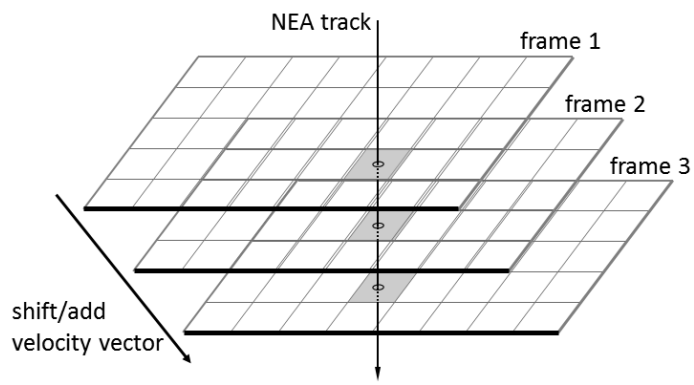

Fig. A.1. Schematic showing the integration of frames by using synthetic tracking. Frames are displaced according to the velocity of a NEO so that it is at the same location in all the frames during the integration (adopted from Shao et al. 2014)

\section{Appendix A: Search for potentially hazardous NEOs with the Synthetic Tracking Technique}

As NEOs are moving against the background stars, their resulting images when using a conventional CCD are streaked, resulting in degradation in sensitivity. The NRC report included a simulation of a hypothetical $2 \mathrm{~m}$ space telescope conducting a NEO search. The optimal integration time for this instrument was only $8 \mathrm{~s}$ with $30 \mathrm{~s}$ of slewing between adjacent FOVs (S. Chesley of JPL 2015, priv. comm.). Longer CCD exposures do not improve sensitivity because of image streaking. The technique of co-adding multiple frames of data to stack the images of faint object was first developed and demonstrated for trans-Neptunian objects (TNO; Bernstein et al. 2004). The images were taken over periods of months. Gural et al. (2005) used the same technique, also called multi-hypothesis matched filter (MHMF), for processing on asteroids with images taken over a period of minutes/hours. Synthetic tracking combines MHMF with faster cameras to detect smaller objects moving at higher angular velocities. This technique makes it possible to achieve a sensitivity down to a $\sim 20.5$ mag object with $10 \mathrm{~cm}$ optics in an 800 s observation, thus offering significant improvements in the sensitivity of detection of NEOs. Below we discuss this technique as it applies for a CubeSat-based version of a synthetic tracking camera.

\section{A.1. Improving sensitivity, $S / N$, and astrometry with synthetic tracking}

Traditional approaches to discovering NEOs relies on CCD exposures of $\sim 30 \mathrm{~s}$. Typically, CCDs require $\sim 10 \mathrm{~s}$ read-out time at low noise $\left(\sim 3 \mathrm{e}^{-}\right)$. Although this approach is effective in detecting slowly moving NEOs, for faster objects it results in a streaked image on the CCD and leads to a significant trailing loss of sensitivity. Intuitively, trailing loss results from the fact that the streaked image distributes photons comprising its signal over a larger area on the CCD (compared to those received from a stationary object) yielding a reduced signal per unit area. There have been many studies on trailing losses. Shao et al. (2014) quantify the trailing loss in $\mathrm{S} / \mathrm{N}$ as a factor $w /(w+s)$, where $w$ is the width of a seeing-limited point-spread function (PSF) and $s$ is the length of the streak. The longer exposure leads to a longer streak length and results in a smaller $\mathrm{S} / \mathrm{N}$.

Compared to the conventional method of a single $30 \mathrm{~s}$ exposure, synthetic tracking uses multiple short exposures: for example, 60 frames at $2 \mathrm{~Hz}$ over the same $30 \mathrm{~s}$ interval. For a small object, a single $0.5 \mathrm{~s}$ exposure image is not sufficient to detect the object in one frame; instead, an addition of appropriately shifted (a) NEA image, current state-of-the-art

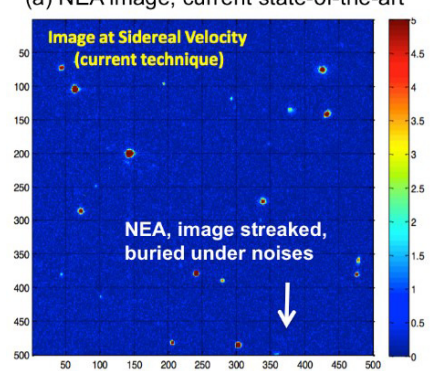

(b) NEA image, synthetic tracking

Fig. A.2. Synthetic tracking images from integrating more than 500 frames taken at $17 \mathrm{~Hz}$ at sidereal velocity a) and velocity of asteroid b) (adapted from Shao et al. 2014). Horizontal axis is the pixel number; vertical axis is the pixel number (left) and the signal intensity (right, in color).

image frames is needed to reconstruct the image of the object. Figure A.1 illustrates the shift/add technique. We shift each subsequent image by an assumed velocity vector. If that assumed velocity is the actual NEO velocity, all the NEO photons will end up in the same pixels in the stacked image. However, for an unknown NEO with an unknown velocity, many different velocities must be tried to determine the true velocity.

NEOs are found by conducting a 4D search in our 3D data cube for $\left(x, y, v_{x}, v_{y}\right)$, which are positions and velocities of NEOs. This effort is computationally intensive (Zhai et al. 2014). For a ground-based facility, the search is done on a graphics-processing unit (GPU) with 2500 cores with a velocity grid of size $100 \times 100$, with velocity grid spacing of $1^{\prime \prime}$ per $30 \mathrm{~s}$. This ensures that the maximum velocity error when searching for NEOs is less than $0.5^{\prime \prime}$ in $30 \mathrm{~s}$, which means that the images are streaked by less than $0.5^{\prime \prime}$ along right ascension (RA) or declination (Dec), which is a negligible trailing loss for $1^{\prime \prime}$ pixels. A typical velocity-searching range covers $\pm 40^{\circ}$ /day in both $\mathrm{RA}$ and Dec, which is adequate for most NEO detections. A maximal velocity of $40^{\circ}$ day is enough to cover over $99.9 \%$ of all NEOs. However, even faster-moving objects will be detected but result in streaked NEO images and therefore lower sensitivity.

We have demonstrated the performance of the synthetic tracking technique towards improving the detection $\mathrm{S} / \mathrm{N}$ by successfully detecting a faint object with an apparent magnitude of 23 ( $H \sim 29.5 \mathrm{mag}$ assuming the asteroid velocity is $10 \mathrm{~km} \mathrm{~s}^{-1}$ detected at 20 lunar distances) on the Palomar $5 \mathrm{~m}$ telescope (Zhai et al. 2014). The object was moving at $\sim 6^{\circ} /$ day, covering $\sim 7^{\prime \prime}$ during the $30 \mathrm{~s}$ observation time. Figure A.2 shows (a) the synthetically tracked images for tracking at the sidereal rate and (b) the asteroid from integrating more than 500 frames taken at $17 \mathrm{~Hz}$ by an $\mathrm{EMCCD}^{13}$ with a negligible read noise when used with an EM gain of 200. Image (a) would be the image detected by using the traditional $30 \mathrm{~s}$ exposures. The asteroid image is a $7^{\prime \prime}$ streak with a surface brightness of a 25 mag star, with a sky background of $\sim 21 \mathrm{mag} /\left({ }^{\prime \prime}\right)^{2}$. We detected this object, shown in (b), with an $S / N \sim 15$.

The detection above is a good example of using the synthetic tracking technique for detection of a previously unknown, small, fast-moving and otherwise undetectable faint object. It also demonstrates the maturity and functionality of our software that is capable of removing detector artifacts, stars and galaxies, as well as identifying false positives.

${ }^{13}$ Electron Multiplying Charge Coupled Device (EMCCD), see details at http://www.emccd.com/what_is_emccd/ 
Synthetic tracking, in general, is much less sensitive to false positives compared to traditional "tracklet" identification of asteroids. With a data cube with $\sim 60$ consecutive images, cosmic rays are easily removed. With single exposure NEO images, galaxies may mimic a slightly streaked image of a NEO. However, with synthetic tracking, the size of the object is minimized when correct velocity is identified. Therefore, galaxies are never mistaken for NEOs in synthetic tracking because the elongation of the image is minimum at zero velocity. In the "tracklet" approach to NEO detection, the image is "thresholded to identify objects". Objects that move linearly across $~ 3-4$ images taken over $\sim 10-30 \mathrm{~min}$ are identified as NEOs. Because each image is "thresholded" to identify objects, stars whose brightness is near the threshold will appear above the threshold in some images and not others. Stellar false positives are not a problem for synthetic tracking, first because we typically have many tens of images in a data cube, and second, at non-zero velocity, faint stars near the threshold will be streaked and be fainter than they would be if the images were co-added with zero velocity.

Synthetic tracking also improves astrometry of NEOs. It accomplishes this in two ways. First, by mitigating the trailing loss, one achieves more precise measurements due to a higher $\mathrm{S} / \mathrm{N}$. Second, it cancels a number of leading error sources that dominate traditional NEO searches, especially those from the ground (Zhai et al. 2014). Thus, in CCD astrometry of a 2D point source, a template $\mathrm{PSF}^{14}$ is fitted to the CCD data. In synthetic tracking astrometry, a moving template is fitted to the $3 \mathrm{D}$ data cube. Using the images from the data cube, neither the asteroid nor the background stars are streaked. Therefore, the image motion from the atmosphere and telescope tracking errors are now common between the NEO and background stars and, thus, cancel for relative astrometry.

Observations from space are less affected from astrometric errors that are present for ground-based observations. Such an advantage allows for longer integration times yielding higher astrometric precision, which is why deploying a synthetic tracking technique on a space telescope is compelling.

\section{A.2. Moderate sensitivity from a small telescope}

In conventional ground-based NEO searches, it does not make sense to take a CCD exposure that exceeds $\sim 30 \mathrm{~s}$. A NEO at a distance of 0.4 au moving $10 \mathrm{~km} \mathrm{~s}^{-1}$ would appear to move relative to background stars by $1^{\prime \prime}$ in $30 \mathrm{~s}$. Thus, for a groundbased telescope with $1^{\prime \prime}$ pixel, $30 \mathrm{~s}$ is close to the optimal exposure time. Longer exposures would not only produce a streak, but they would also increase the background noise contribution without increasing the signal. On the other hand, with synthetic tracking, we can observe for a much longer time, $T$, than $30 \mathrm{~s}$ with increased $\mathrm{S} / \mathrm{N}$ as $\sqrt{T}$.

The sensitivity of a synthetic tracking camera depends on a number of parameters: telescope diameter, pixel size and the total observation time, assuming the individual exposures are short enough that the motion of the NEO is less than 1 pixel.

Table A. 1 shows the sensitivity of a $10 \mathrm{~cm}$ synthetic tracking camera at $1 \mathrm{au}$. In calculating these sensitivities, we used the $\mathrm{QE}(\lambda)$ of a commercial sCMOS detector ${ }^{15}$ that peaks at $82 \%$ at $0.55 \mu \mathrm{m}$. The QE was multiplied by a $5800 \mathrm{~K}$ black

\footnotetext{
14 The point spread function (PSF) describes the response of an imaging system to a point source or point object.

${ }^{15}$ For details of the detector, please see: http://WwW . hamamatsu . com/jp/en/product/category/5000/5005/C11440-22CU/ index.html
}

Table A.1. Parameters used to estimate sensitivity of synthetic tracking camera.

\begin{tabular}{|c|c|c|}
\hline & Value & Unit/notes \\
\hline Input values: & & \\
\hline Nominal $H$ magnitude & $H=22 \mathrm{mag}$ & size: $\sim 140 \mathrm{~m}$ \\
\hline NEO limiting magnitude & 20.49 & \\
\hline NEO distance & 0.362 & $\mathrm{au}$ \\
\hline Transverse velocity & 12 & $\mathrm{~km} \mathrm{~s}^{-1}$ \\
\hline Phase angle & 0 & deg \\
\hline Telescope diameter & 100.0 & $\mathrm{~mm}$ \\
\hline Total QE & 0.64 & \\
\hline Pixel size & 3.30 & $\prime \prime$ \\
\hline Detector read noise & 1.20 & $\mathrm{e}^{-}$ \\
\hline Frame time & 10.00 & $\mathrm{~s}$ \\
\hline Total integration time & 800.00 & $\mathrm{~S}$ \\
\hline Total FOV & 14.10 & $\left({ }^{\prime \prime}\right)^{2}$ \\
\hline Sky background & 22.0 & $\operatorname{mag} /\left({ }^{\prime \prime}\right)^{2}$ \\
\hline $0 \mathrm{~V}$-mag reference & $2.48 \times 10^{10}$ & phot $/ \mathrm{m}^{2} / \mathrm{s}$ \\
\hline Derived values: & & \\
\hline Apparent magnitude & 20.49 & mag \\
\hline Flux detected & 0.72 & $\mathrm{e}^{-} / \mathrm{s}$ \\
\hline Noise/frame variance & 83.86 & $\mathrm{e}^{-}$ \\
\hline Signal/frame & 7.17 & $\mathrm{e}^{-}$ \\
\hline Total S/N & 7.00 & in $800 \mathrm{~s}$ \\
\hline
\end{tabular}

Notes. Total QE describes a combined QE, which includes the system's optical throughput and detector QE; otherwise detector QE is used.

body emission where 0.0 mag represented a flux of $9.66 \times$ $10^{9} \mathrm{phot} / \mathrm{m}^{2} / \mathrm{s}$ into a $0.089 \mu \mathrm{m}$ bandpass. This resulted in a detected flux of $2.48 \times 10^{10} \mathrm{phot} / \mathrm{m}^{2} / \mathrm{s}$ when integrated from $\sim 0.45-$ $0.9 \mu \mathrm{m}$ for a $0.0 \mathrm{mag} \mathrm{NEO}$, which was assumed to have a solar like spectrum. While it would be reasonable to assume an optics efficiency of $\sim 80 \%$, to be conservative we assumed $55 \%$. We assumed the detector read noise to be $1.2 \mathrm{e}^{-}$and the zodi background at 1 au to be $22 \mathrm{mag} /\left({ }^{\prime \prime}\right)^{2}$. The detector dark current at $0.006 \mathrm{e}^{-} / \mathrm{pix} / \mathrm{s}$ at $-30{ }^{\circ} \mathrm{C}$ was ignored. The zodi background calculated was based on a solid angle that was calculated using the following information: the pixel size, the optical PSF of a Canon $400 \mathrm{~mm} \mathrm{f} / 4$ lens with a $3.6^{\prime \prime}$ spot size in reflected sunlight, as assumed jitter of $1^{\prime \prime}$ in the spacecraft and a factor that is due to the PSF that can straddle more than one pixel. A commercial camera lens is designed to be focusable from $4 \mathrm{~m}$ to infinity. A custom lens designed only for focus at infinity could possibly be slightly better. The long integration time means that a NEO will move across several pixels and more or less uniformly sample the pixel. The effective background was calculated using a simulation of a moving object and a matched filter. The effective zodi background is from a $6.4^{\prime \prime}$ box, not quite $2 \times 2$ pixels when all the effects above are included (pixel size, pixel straddling, optical PSF and diffraction PSF).

If a camera moves closer toward the Sun, the relevant zodi background increases. In this regard, our simulation includes the noise contribution from the dust in the inner solar system by accounting for its intensity variation as a function of heliocentric distance (Roach \& Gordon 1973; Leinert et al. 1982). 


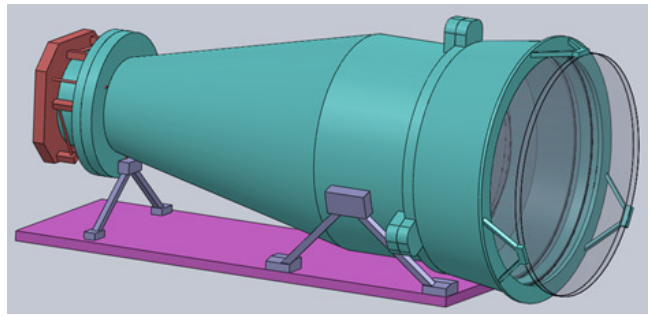

Fig. B.1. A CAD design for the synthetic tracking camera for a CubeSat.

Compared to ground based telescopes, the numbers in Table A.1 may seem optimistic. However, many telescopes are not located at the darkest sites and even at the darkest sites, $21.5 \mathrm{mag} /\left({ }^{\prime \prime}\right)^{2}$ sky background is possible only at new moon. When the sky background due to a full moon gets to $18 \mathrm{mag} /\left({ }^{\prime \prime}\right)^{2}$, the loss in sensitivity ( $\sim 2 \mathrm{mag}$ ) makes the search almost impossible.

\section{Appendix B: Emerging capabilities of interplanetary CubeSats}

There is an on-going paradigm shift occurring in the satellite industry that may be compared to events dating back more than three decades to when personal computers disrupted mainframe computing. Small spacecraft, and their most popular subclassification, CubeSats, have tremendous potential, not only in the commercial realm, but also by innovating established space programs through the use of CubeSats for research and technology development and demonstration.

The low-cost CubeSat components, shorter development cycle, and availability of frequent launch opportunities for smaller satellites make it quicker and less expensive to get the latest capabilities into space. JPL is involved in these efforts with several CubeSat projects both in LEO and for deep space, with several already launched and many more being developed ${ }^{16}$.

\section{B.1. System design: science instrument}

The synthetic tracking camera is the primary instrument for the NEO search (Fig. B.1). The lens would be similar to commercially available camera lenses. The optics and structure would be designed to survive launch loads and will have the necessary thermal insulation to passively cool the instrument to maintain the optics temperature stable to within $5{ }^{\circ} \mathrm{C}$ of the desired value.

The sensor is a $4 \mathrm{~K} \times 4 \mathrm{~K}$ class $\mathrm{sCMOS}^{17}$ detector. Second generation sCMOS detectors have low $\sim 1.2 \mathrm{e}^{-}$read noise and low $<0.1 \mathrm{e}^{-} / \mathrm{pix} / \mathrm{s}$ dark current. The plate scale of the camera $\sim 3.3^{\prime \prime} /$ pix, which sets the amount of zodi background per pixel.

\footnotetext{
${ }^{16}$ For JPL's effort in the CubeSat area, please visit http: //cubesat.jpl.nasa.gov/. The NASA efforts in this area are summarized in http://www.nasa.gov/directorates/spacetech/ small_spacecraft

${ }^{17}$ For details on Scientific CMOS (sCMOS) cameras, please visit http://www . andor.com/scientific-cameras/

neo-and-zyla-scmos-cameras. Larger pixel format sCMOS detectors with $16 \mathrm{Mpix}$ currently under development and are expected to be available in 2017.
}

\section{B.2. Observation cadence and data processing}

A crucial capability for using synthetic tracking is to handle onboard data processing in real-time, which we now discuss. Our nominal observation uses exposure times of $10 \mathrm{~s}$ and $800 \mathrm{~s}$ integration time per field ( 80 frames). The detection threshold of $\mathrm{S} / \mathrm{N}$ is set to 7 . The slew time for moving from one field to another field is $10 \mathrm{~s}$. With a FOV of $14\left(^{\circ}\right)^{2}$, the sky can be scanned approximately for $\sim 13$ days if we adopt the same approach as NEOCam to restrict the ecliptic latitude range to $\pm 45^{\circ}$ and with sun exclusion angle beginning at $\sim 60^{\circ}$. To determine the orbit of the detected asteroids, the observations of the same asteroid need to be connected. In Sect. 4, we will discuss how to arrange the observation cadence to make the connection of observations feasible. For most of our discussions and the case studies presented in Sect. 3, we assume this simple sky scanning scenario.

We process our basic data set, which contains 80 images of size $(4 \mathrm{~K} \times 4 \mathrm{~K})$, in real-time (within $810 \mathrm{~s}$ ) using fieldprogrammable gate array (FPGA). The main computation load comes from the synthetic tracking search, where we integrate 80 frames for each velocity vector in a two-dimensional (RA, Dec) velocity grid with spacing $\sim 2$ pixels per $800 \mathrm{~s}\left(\sim 0.2^{\circ} /\right.$ day $)$. Because NEAs are typically detected at $\sim 0.36$ au with a typical velocity of $10 \mathrm{~km} \mathrm{~s}^{-1}$, moving at $\sim 1^{\circ} /$ day, it is sufficient to search over a range of $\pm 6^{\circ} /$ day, giving a velocity grid of size $(60 \times 60)$. The total amount of computations needed is estimated as $(4000)^{2} \times 80 \times(60)^{2}$ per $810 \mathrm{~s}$ or 5.7 GFLOPS. The signals detected by FPGA is then further analyzed by a four-parameter (2D location and velocity) least-squares fitting, which provides a better estimate of the $\mathrm{S} / \mathrm{N}$ of the detected signal and more precise astrometry.

It is relevant to discuss the possibility of processing $800 \mathrm{~s}$ of data that consists of two sets of 40 frames separated by $2 \mathrm{~h}$ without adding significant extra computation load. This is particularly useful for observation cadences supporting linkage of observations for orbit determination to be discussed in Sect. 4. A direct synthetic tracking search would require a velocity grid with a very fine resolution of 2 pixels per $2 \mathrm{~h}\left(\sim 0.022^{\circ} /\right.$ day $)$ because the total temporal range of the data set is $2 \mathrm{~h}$. To cover a velocity range of $\pm 6^{\circ} /$ day, we need a velocity grid of size $540 \times 540$ giving $(4000)^{2} \times 80 \times(540)^{2}$ operations per data cube $(800 \mathrm{~s})$ or 467 GFLOPS to perform the synthetic tracking search.

We introduce a two-stage synthetic tracking to greatly reduce the amount of computations needed: 1) We perform synthetic tracking using a velocity grid with spacing of 2 pixels per $800 \mathrm{~s}$ to separately integrate the first and second 40-frames to form two synthetic images; and 2) we combine the two synthetic images using a fine velocity grid with spacing of 2 pixels per $2 \mathrm{~h}$. For integrating $400 \mathrm{~s}$ data, it is sufficient to use a velocity grid with spacing of 2 pixels per $800 \mathrm{~s}\left(\sim 0.2^{\circ} /\right.$ day $)$. A grid of size $(60 \times 60)$ covers the range of $\pm 6^{\circ} /$ day. We estimate the amount of computation needed for the first stage to be $(4000)^{2} \times 40 \times 2 \times(30)^{2}$ per data cube ( $820 \mathrm{~s}$, note there is one slew per $400 \mathrm{~s}$ observation), or 5.6 GFLOPS. For the second stage, the amount of computation $(4000)^{2} \times 2 \times(540)^{2}$ operations per data cube or $\sim 12$ GFLOPS. We can further reduce the amount of the computation needed for the second stage by performing synthetic tracking only for pixels of interest.

Note that for a signal of $S / N=7$ from integrating 80 frames, over the first 40 frames, we expect the $\mathrm{S} / \mathrm{N}$ to be $\sim 5$. We, therefore, can require that only pixels with $S / N>3$ (using a lower threshold than 5 mitigates miss detection due to statistical fluctuation of the signal strength) from integrating the first 40 frames to be processed. This greatly reduces the amount of pixels to be 
considered for synthetic tracking by a factor of $\sim 700$ (assuming Gaussian statistics). Thus the amount of computation needed for the second stage becomes $\sim 16$ MFLOPS. So the total computation needed for synthetic tracking search is still $\sim 5.7$ GFLOPS.

\section{B.3. Spacecraft design}

\section{B.3.1. LEO flight system from JPL's Team Xc}

A recent JPL's TeamXc study, a rapid concurrent design engineering session, developed a detailed design for a Low Earth Orbit (LEO) CubeSat with synthetic tracking (Zarifian et al. 2014). The LEO flight system design relied on a 6U CubeSat architecture comprised largely of commercial components with flight heritage from previous CubeSat missions that have flown in LEO. Here we describe all major components of the system, except for the telecommunication, navigation, and propulsion systems; these systems were augmented for the interplanetary application due to critical differences for an interplanetary destination and are discussed in the following section.

The avionics of the spacecraft will include the radiationtolerant LEON processor ${ }^{18}$ and algorithms to perform data analysis, command, and control. The computational requirements are dominated by multi-vector shift/add processing, which is required for the synthetic tracking algorithms. A top-level conceptual design of the computation architecture was studied using flight-qualified FPGAs. As discussed in Appendix B.2, the processor requirement is $\sim 5.7$ GFLOPS, which could be done by programming 24 computational units into a single flightqualified FPGA. In addition to the FPGA, approximately $6 \mathrm{~GB}$ of RAM would also be needed to store the data for subsequent on-board processing. Approximately $10 \%$ of the arithmetic processing capabilities of a Virtex-7 FPGA would be needed. The FPGA power consumption is anticipated to be $\sim 6-7 \mathrm{~W}$.

The attitude control system will be based on the Blue Canyon Technologies XACT ${ }^{19}$ unit, which consists of a star tracker, reaction wheels, and an inertial measurement unit (IMU), to achieve the pointing stability and agility requirements of the mission. Based on the specifications, the XACT unit achieves pointing control of $10.8^{\prime \prime}(1 \sigma)$, knowledge of $6^{\prime \prime}(1 \sigma)$ and stability of $10.8^{\prime \prime}$ over $4 \mathrm{~s}$. The NEO CubeSat requires a pointing control at $2^{\circ}$ which is easily met by XCAT. However, the pointing stability requirement for NEO CubeSat is $6^{\prime \prime}$ over $4 \mathrm{~s}$, which is at the limit for the current XACT unit. Thus, some modification may be required to meet the pointing stability requirements once improved thermal/structural designs are available and an end-to-end simulation can be done. Such a modification may lead to additional isolation for jitter mitigation, which can be achieved by adding a fourth reaction wheel or other stabilization methods.

A CubeSat reaction wheel, such as model RW8 by Blue Canyon technologies, has a max torque of $0.007 \mathrm{Nm}$, weighs $250 \mathrm{~g}$ and can slew a $10 \mathrm{~kg}$ spacecraft $(30 \mathrm{~cm}$ max dimension) by $4^{\circ}$ in $<7 \mathrm{~s}$. We use $10 \mathrm{~s}$ as a "typical" time to slew the telescope between adjacent FOVs.

The power system consists of i) deployed solar arrays that generate $\sim 40 \mathrm{~W}$ at the beginning of the mission; ii) a solar array drive assembly to point the arrays at the Sun; iii) the onboard battery to support high-powered events; and iv) a power

\footnotetext{
${ }^{18}$ For details on LEON processors: http://www . gaisler.com/

${ }^{19}$ For details on the Blue Canyon Technologies XACT unit: http://bluecanyontech.com/product/xact/. Also, for XACT, High Performance Attitude Control for CubeSats, see

http://bluecanyontech. com/wp-content/uploads/2012/07/ BCT-XACT-datasheet-1.5.pdf
}

management system. A standard aluminum CubeSat structure will protect components from radiation in the LEO environment. A standard deployment system will house and deploy the spacecraft.

The total current best estimate for a "dry" mass of the LEO system was estimated to be $8.2 \mathrm{~kg}$, without margin (Zarifian et al. 2014). The cost, including mission and science operations, was found to be $\sim \$ 9 \mathrm{M}$, including $20 \%$ margin.

\section{B.3.2. Computing architecture}

In developing the required computing architecture, we account for the fact that an average $H=22$ mag NEO can be detected by a $10 \mathrm{~cm}$ synthetic camera at a distance of $\sim 0.36 \mathrm{au}$, when the observatory is at $\sim 0.9$ au from the Sun. If the NEO moves with a velocity of $12 \mathrm{~km} \mathrm{~s}^{-1}$ relative to the observatory, its angular motion is $\sim 0.046^{\prime \prime} / \mathrm{s}$. Since the pixel is $3.3^{\prime \prime}$, we have to record images faster than one per $100 \mathrm{~s}$ for the streak related loss in sensitivity to be small, for the total $800 \mathrm{~s}$ of observation. We have chosen $10 \mathrm{~s}$ exposures to capture i) NEOs with higher velocity and ii) smaller NEOs that can only be detected at ranges much closer than $\sim 0.36$ au. A $800 \mathrm{~s}$ observation would have 80 images. When we shift/add these images, we should shift and add with a velocity vector range of velocities that includes the highest velocities a NEO can have and the spacing between velocity vectors should result in a velocity mismatch of less than 2 pixel/800 s data cube.

At a high level, the on-board data processing computer performs four core functions: data reduction and star removal, integer shift-and-add, candidate selection, and postage-stamp image generation for down-link. The shift-and-add operation provides all driving requirements, as it applies two orders of magnitude more arithmetic operations than data reduction and star removal, and the final two steps operate on significantly smaller datasets.

Figure B.2 outlines our proposed FPGA-based computational architecture. We have baselined a Xilinx Virtex-7 device ${ }^{20}$, which is currently slated to fly on the NASA TESS mission ${ }^{21}$. Camera data stream into the FPGA and are offloaded into one of the two separate external memory banks, which operate as a ping-pong buffer pair. Once an entire datacube is collected, data collection immediately resumes and targets the second memory bank. Data processing is performed as described in the previous section, with candidate objects reported to an on-board softcore microprocessor. The processor runs software to identify unique objects among the candidates and coordinates postage stamp collection and downlink to Earth.

For this initial design, we have chosen 3 GB of 64-bit DDR3 at $200 \mathrm{MHz}$ per external memory bank, which supports a data rate of 12.8 GBps. By utilizing a parallel NEO detection algorithm with thirty separate streams running at $200 \mathrm{MHz}$ and $1 \mathrm{op}-$ eration per cycle, the system provides 6 GFLOPS.

A prototype implementation including memory controllers, shift-and-add logic, softcore microprocessor, and on-board memory has been built and run through FPGA design/simulation tools. The multivector-shift/add part of the processing uses $\sim 5 \%$ of the arithmetic units available on Virtex-7. This design was also analyzed with Xilinx's power estimation tool, with a resultant power draw of approximately $6.5 \mathrm{~W}$ split equally among configuration logic, device I/Os, and design logic.

\footnotetext{
${ }^{20}$ For more information on Xilinx Virtex-7 devices, please see: http://wWw . xilinx.com/products/silicon-devices/fpga/ virtex-7.html

21 For information on the NASA's Transiting Exoplanet Survey Satellite (TESS) mission, please visit http://tess.gsfc.nasa.gov/
} 


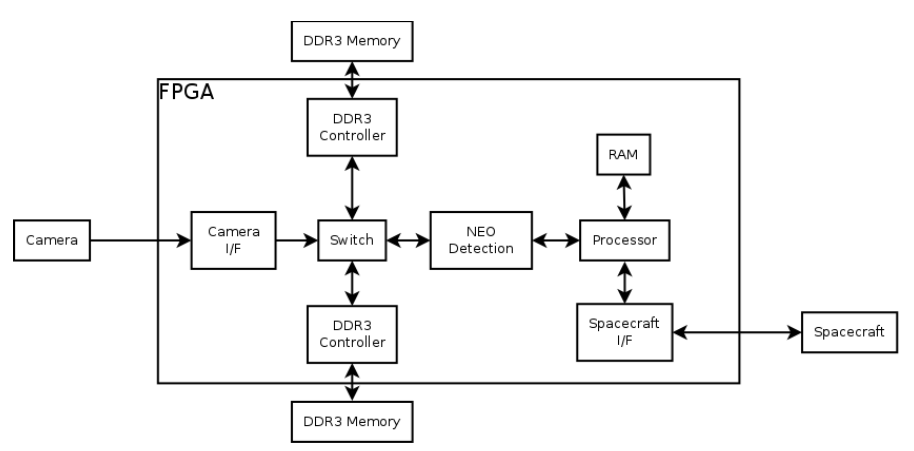

Fig. B.2. Proposed FPGA-based computational architecture.

\section{B.3.3. Required changes from the LEO to interplanetary NEO SmallSat design}

The interplanetary application of the NEO SmallSat requires changes to the LEO CubeSat design discussed above. The required changes to the hardware may be inherited from many common spacecraft elements developed for interplanetary CubeSat missions that have been or are currently being developed at JPL. These missions include INSPIRE ${ }^{22}$, LunarFlashlight, NEAScout, and $\mathrm{MarCO}^{23}$, which are expected to fly before this NEO SmallSat, providing additional maturity and flight heritage to our design. The major changes for the interplanetary CubeSat design will affect several subsystems including navigation, communication, and propulsion, as summarized in Table B.1.

To enable navigation and telecommunication in deep space, the interplanetary NEO SmallSat will include the Iris transpon$\operatorname{der}^{24}$ (Duncan et al. 2014) and a High Gain Antenna (HGA), such as the one used on the NEAScout mission ${ }^{25}$. With the Iris transponder and HGA with direct Earth transmission to the NASA's Deep Space Network (DSN), data rates of approximately $1 \mathrm{kbps}$ will be achievable at the closest expected distance of 0.3 au (once the constellation is established), data rates of $\sim 90 \mathrm{bps}$ are achievable at distances of $1 \mathrm{au}$, and less than $30 \mathrm{bps}$ at the maximum distance of 2 au. Throughout the mission, the data rates will be adjusted according to the spacecraft distances from Earth. To return the total of approximately $128 \mathrm{MBytes}$ of data throughout the $4 \mathrm{yr}$ mission with six SmallSats, each spacecraft will download for $0.1 \%$ or $16.4 \mathrm{mins} /$ week (at $0.3 \mathrm{au}$ ) and $7 \%$ or $12.2 \mathrm{~h}$ per week (at $2 \mathrm{au}$ ) of the mission time, which is accounted for in the engineering time allocation. In future studies we will also investigate the option of sending data through inter-satellite relays to return the data to Earth to achieve higher throughput.

Synthetic tracking is sensitive to "moving" objects, especially to those that move over $3^{\prime \prime}$ in $400 \mathrm{~s}$. The neo. jpl .nasa. gov site lists $\sim 100$ "near" encounters of known asteroids. For the majority of these EGs (i.e., 98-99\%) their geocentric velocity is larger than $3 \mathrm{~km} \mathrm{~s}^{-1}$. Synthetic tracking is immune to false positives from cosmic rays, galaxies, and stars near the detection threshold of $7 \sigma$ (see Appendix A.1 for discussion).

\footnotetext{
${ }^{22}$ For information in INSPIRE Mission: http://cubesat.jpl. nasa.gov/projects/inspire/overview.html

${ }^{23}$ For information on MarCO mission: http://space. skyrocket. de/doc_sdat/marco.htm

${ }^{24}$ http://mstl.atl.calpoly.edu/ bklofas/Presentations/ DevelopersWorkshop2014/Duncan_Iris_Deep_Space_

Transponder.pdf

${ }^{25}$ For details on the NEA-Scout (Near Earth Asteroid Scout) is a CubeSat mission: http://space.skyrocket.de/doc_sdat/ nea-scout.htm
}

Table B.1. Cost estimate for the interplanetary version of NEO SmallSat resulting from changes to a LEO CubeSat design.

\begin{tabular}{|c|c|c|}
\hline Change & Function & Cost, $\$ \mathrm{M}$ \\
\hline \multicolumn{2}{|c|}{ Total LEO CubeSat (before margins) } & 7.5 \\
\hline $\begin{array}{l}\text { Replacement of UHF ra- } \\
\text { dio with Iris deep-space } \\
\text { Transponder }\end{array}$ & $\begin{array}{l}\text { To enable interplanetary } \\
\text { communication and navi- } \\
\text { gation }\end{array}$ & 0.4 \\
\hline $\begin{array}{l}\text { Addition of propulsion } \\
\text { system }\end{array}$ & Busek Thrusters $(4 \mathrm{x})$ & 1.0 \\
\hline $\begin{array}{l}\text { Additions to ACS, power, } \\
\text { structure, thermal subsys- } \\
\text { tems }\end{array}$ & $\begin{array}{l}\text { Increased spacecraft size, } \\
\text { mass, and design work ex- } \\
\text { pected }\end{array}$ & 0.5 \\
\hline Operations & $\begin{array}{l}\text { Trajectory planning and } \\
\text { ground systems }\end{array}$ & 1.0 \\
\hline \multirow{2}{*}{\multicolumn{2}{|c|}{$\begin{array}{r}\text { Total change } \\
\text { Total Interplanetary NEO SmallSat } \\
\text { (before margins) }\end{array}$}} & 2.9 \\
\hline & & 10.4 \\
\hline \multicolumn{2}{|c|}{$\begin{array}{r}\text { Total Interplanetary NEO SmallSat } \\
\text { (including } 20 \% \text { margin) }\end{array}$} & 12.5 \\
\hline
\end{tabular}

Although the Gaia mission can detect $\gtrsim 60-80 \%$ of NEOs, it is capable of detecting all the main belt asteroids brighter than $V=20$ (or $\sim 2 \mathrm{~km}$ in size, see Tanga \& Mignard 2012). Should we detect a moving object from the Gaia catalog, we will not downlink the corresponding 125 pixel postage stamp of that object nor will we follow it up with observations $2 \mathrm{~h}$ later. This minimizes the down-link data to 20000 NEOs brighter than $H=22$ mag. Our simulations show that, on average, each of these NEOs will be detected approximately ten times during the $3.5 \mathrm{yr}$ mission. The ultimate telecom system for our mission would allow for this anticipated data volume. Fitting orbits with only ten detections is challenging, but some progress in this area has been made (Granvik \& Muinonen 2005; Granvik et al. 2007; Granvik \& Muinonen 2008); we will use these techniques in the future.

To send the SmallSat into a solar orbit at heliocentric ranges of less than 1 au, a dedicated propulsion system is required. Compared to the ground-based observations or a LEO version, the solar orbit would provide the mission with a better observing geometry and a faster orbit to conduct NEO/EG search. The CubeSat would share a ride to GEO and then it would use an extra propulsion motor to enter solar orbit. Although there are no currently existing CubeSat-class propulsion modules with the required capabilities, several promising propulsion options are available, including the Busek Electrospray Thrusters (BET) ${ }^{26}$ and chemical thrusters built by Aerojet Rocketdyne $^{27}$. There are other propulsion options based on the systems that are currently being developed. In the near future these new systems will be able to satisfy the size, power, thermal, and launch constraints of a small spacecraft and should be considered in an end-to-end system optimization (Mueller et al. 2010; Marrese-Reading et al. 2010; Spangelo et al. 2015).

We also expect changes to the power, thermal, structural, and attitude control systems. These changes will result in increases in mass, volume, power, and cost estimates for the EG SmallSat, as reflected in Table B.1. The highest power for the

\footnotetext{
26 For details on the Busek Electrospray Thruster system, please visit http://busek.com/technologies_espray.htm

27 For details on the Aerojet Rocketdyne thruster, http://www . rocket. com/cubesat
} 
Table B.2. Current best estimates (CBE) for the mass of the interplanetary NEO SmallSat.

\begin{tabular}{|c|c|c|}
\hline Subsystem & Components & Mass, $\mathrm{kg}$ \\
\hline Science instrument & Synthetic tracking camera & 2.8 \\
\hline $\begin{array}{l}\text { Navigation, communica- } \\
\text { tion }\end{array}$ & $\begin{array}{l}\text { Iris transponder; high gain } \\
\text { antenna }\end{array}$ & 1.0 \\
\hline Command, data handling & LEON processor and board & 0.25 \\
\hline Attitude control system & $\begin{array}{l}\text { BCT XACT with sized-up } \\
\text { wheels }\end{array}$ & 0.85 \\
\hline Propulsion & Busek thrustes $(4 \mathrm{x})$ & 4.8 \\
\hline Thermal & Radiator & 1.0 \\
\hline Power & Solar panels, batteries, EPS & 0.86 \\
\hline Mechanical & Structure & 2.0 \\
\hline \multirow{2}{*}{\multicolumn{2}{|c|}{$\begin{array}{r}\text { Total Dry } \\
\text { Total Wet (without margin) }\end{array}$}} & 13.2 \\
\hline & & 22.2 \\
\hline
\end{tabular}

interplanetary CubeSat is expected to be approximately $47 \mathrm{~W}$ during the download mode. To accommodate these changes we would have to resize the solar panels and/or batteries. Trajectory planning and ground operations are also expected to result in additional cost increases. Although pointing requirements are similar to the LEO version, we expect to use larger reaction wheel assemblies (RWA) which may result in larger torques on the spacecraft during RWA de-saturation and motivate changes to the algorithms and thruster controls during RWA de-saturation periods. Additional spacecraft structural thickness will be implemented surrounding the radiation-sensitive components such as components of the attitude control system (ACS; i.e., the stellar reference unit (SRU), RWA driver) and the electrical power subsystem (EPS). The small spacecraft community is developing other safety and mission assurance strategies to mitigate these concerns for interplanetary missions (Fazio et al. 2014).

The resulting spacecraft will be a $9 \mathrm{U}$ CubeSat bus. The cost estimate for the interplanetary NEO CubeSat is $\$ 12.5 \mathrm{M}$, including a $20 \%$ margin, as shown in Table B.1. We start with a cost estimate for the LEO CubeSat provided by the JPL Team Xc and add our estimates for the cost changes for each of the affected subsystems. Table B.1 shows the cost estimate for a spacecraft that could be used for a NEO SmallSat constellation. It shows that a constellation capable of detecting $90 \%$ of EGs would be 5-15 times less expensive than the missions/facilities considered in the 2010 NRC report. A more detailed mission design study would be needed to refine the cost and will be done in the near future.

\section{B.4. Spacecraft mass and power budgets}

The CubeSat is expected to have a dry mass of approximately $13.2 \mathrm{~kg}$, including payload and all major subsystems power, propulsion, command and data handling, attitude determination and control, thermal, and structure. Table B.2 shows the current best estimate (CBE) for the masses of each subsystem. The required propellant mass will depend on the orbit chosen for the spacecraft, and is expected to be $\sim 9 \mathrm{~kg}$ (to boost to an orbit of $\sim 0.8 \mathrm{au}$ ). Therefore, the total expected wet mass is approximately $22.2 \mathrm{~kg}$ before margins, representing realistic mass allocation for a SmallSat to be flown on an interplanetary mission.

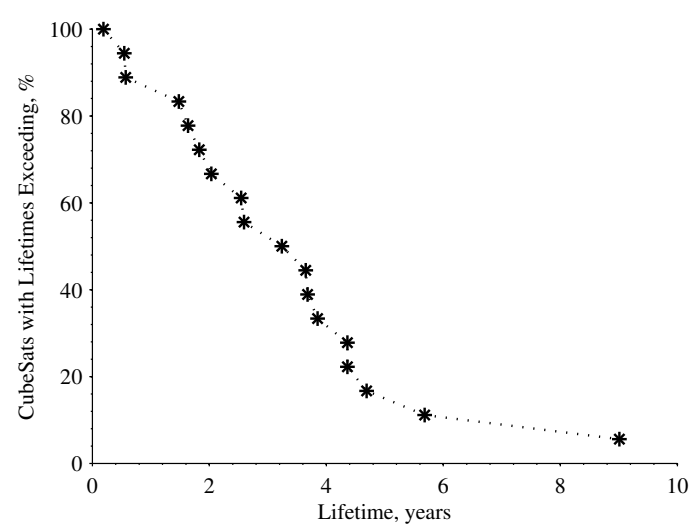

Fig. B.3. Lifetime Statistics from historic LEO CubeSat missions (filtered for unlikely failures).

\section{B.5. Spacecraft lifetimes and constellation architectures}

The ability to achieve the mission's science objectives of detecting $90 \%$ of all the EGs is directly related to the mission lifetime; therefore, we are concerned about CubeSat failures. We have studied historical data on CubeSat failures, available for missions developed and launched by universities, government, and industry. Figure B.3 shows the statistics for typical lifetimes of over 200 CubeSats (including 1U, 2U, 3U) launched since 2003, where the full data set is described by Fazio et al. (2014). We closely examined the data and filtered out failures due to launch or deployment, and those due to causes that are expected to be preventable such as communication or power problems due to poor designs, or latch-up due to potential radiation exposure.

We also filtered out CubeSats that de-orbited, where they did not fail due to technical reasons, and those that were only recently launched and have not yet failed. Most of this historical data is based on university-built CubeSats, so using this data to inform expected lifetimes is conservative as the proposed CubeSats will be developed by professional engineers using highheritage components. Furthermore, there is no available statistical information about planetary CubeSats because they have not yet been launched, so this is the only data that we can use to extrapolate performance. Assuming a Gaussian distribution, the lifetime is $\approx 2.1 \mathrm{yr}$. Based on our statistics, $\approx 45 \%$ of CubeSats have lifetimes over $3.5 \mathrm{yr}$, as shown in Fig. B.3. This indicates the trend in technological maturity and growth of the lifetimes of the CubeSats. Two MarCO CubeSats ${ }^{28}$ have been completed, and are waiting for launch. They are designed to piggy back on a ride to Mars and act as communication relay satellites.

\section{B.6. Setting up a constellation of SmallSats}

A detailed mission analysis that optimizes the mass and time of setting up a NEO SmallSat constellation, and associated cost is beyond the scope of this paper. Here we take a simplified approach to describe a sequential transfer of several SmallSats from an Earth's orbit to a 0.8 au orbit. We start with the first spacecraft. A spacecraft in a 0.8 au orbit has a period of 261 days. After $\sim 2.5 \mathrm{yr}$, it will have traveled around the Sun $\sim 1260^{\circ}$, while the Earth will have traveled $\sim 900^{\circ}$ around the Sun. A low cost way to deploy a constellation of six SmallSats to a heliocentric orbit would be to send them out from the Earth's orbit every

${ }^{28}$ For more information on MarCO CubeSats, pelase see http: //www . jpl.nasa.gov/cubesat/missions/marco.php 
$\sim 152$ days. The time between the 1 st of the six spacecraft and the last would be $\sim 2.1 \mathrm{yr}$.

For constellation orbits close to $1 \mathrm{au}$, for example, $0.95 \mathrm{au}$, this method of "setting up" a constellation can be time consuming. With an additional propulsion, one could enter an orbit with a smaller semi-major axis of say, $0.8 \mathrm{au}$, wait for the spacecraft to race ahead of Earth, then fire the thruster to get back to $0.95 \mathrm{au}$. Most $(\sim 70 \%)$ of the $9 \mathrm{~kg}$ of propellant mentioned in Appendix B.4 is used to escape Earth's gravity (from LEO), so more complex orbit maneuvers would not be prohibitively expensive.

Since the SmallSats can observe as they transit to the 0.8 au orbit, the transfer time could, in principle, lengthen the mission lifetime by only $\sim 1.1 \mathrm{yr}$. The simulation of six 9U CubeSat in Sect. 3 showed that $\sim 3.8$ yr of observations could detect $90 \%$ of $H=22$ mag NEOs. Therefore, at least in theory, the survey could be conducted in $\sim 5 \mathrm{yr}$ after the 1st spacecraft leaves the Earth's orbit. Our preliminary analysis has shown that the Congressionally mandated NEO survey goals may be accomplished in $\sim 5 \mathrm{yr}$ by a constellation costing a small fraction of the missions studied in (NRC 2010). This is the unique advantage of a SmallSat-based mission architecture, which enables trade-offs to be made regarding the survey completion time, risk, and cost.

The (NRC 2010) report concluded that it would take approximately ten years for a single spacecraft with a $50 \mathrm{~cm}$ telescope to conduct a search for $90 \%$ of $H=22$ mag NEOs. Designing a spacecraft with the redundancy and required testing to ensure survival for ten years can significantly increase the mission cost. With a constellation of six SmallSats, a failure of a single spacecraft is not catastrophic; in fact, the constellation's performance degrades gracefully with respect to loosing a node. Figure 4 shows the sensitivity in search time to constellation size. Given the low cost of an additional SmallSat, one may consider placing more than six spacecraft at the desired solar orbit to form a redundant constellation. Such a redundancy would not only reduce the time of conducting the $90 \%$ NEO survey, it would also reduce the risk of the NEO search to potential spacecraft failures identified in (NRC 2010). 